\title{
Channel flow of a tensorial shear-thinning Maxwell model: Lattice Boltzmann simulations
}

\author{
S. Papenkort ${ }^{1,2, a)}$ and Th. Voigtmann ${ }^{1,2}$ \\ ${ }^{1}$ Zukunftskolleg and Fachbereich Physik, Universität Konstanz, 78457 Konstanz, Germany \\ ${ }^{2}$ Institut für Materialphysik im Weltraum, Deutsches Zentrum für Luft- und Raumfahrt (DLR), \\ 51170 Köln, Germany
}

(Received 29 January 2014; accepted 10 April 2014; published online 25 April 2014)

\begin{abstract}
We discuss pressure-driven channel flow for a model of shear-thinning glass-forming fluids, employing a modified lattice-Boltzmann (LB) simulation scheme. The model is motivated by a recent microscopic approach to the nonlinear rheology of colloidal suspensions and captures a nonvanishing dynamical yield stress and the appearance of normal-stress differences and a flow-induced pressure contribution. The standard LB algorithm is extended to deal with tensorial, nonlinear constitutive equations of this class. The new LB scheme is tested in 2D pressure-driven channel flow and reproduces the analytical steady-state solution. The transient dynamics after startup and removal of the pressure gradient reproduce a finite stopping time for the cessation flow of yield-stress fluids in agreement with previous analytical estimates. () 2014 AIP Publishing LLC. [http://dx.doi.org/10.1063/1.4872219]
\end{abstract}

\section{INTRODUCTION}

Flow problems involving dense fluids are ubiquitous in nature and industry. ${ }^{1,2}$ They often probe the nonlinearresponse regime, as for example in shear-thinning fluids, where the effective viscosity decreases rapidly with increasing shear rate and hence depends sensitively on the flow geometry. Since such flows are no longer described by the standard Newtonian linear-response behavior where shear stress and shear rate are linearly related, they are termed nonNewtonian.

Ultimately, one would like to understand the microscopic mechanisms of non-Newtonian fluids and how these determine the macroscopic flow properties. This is a formidable task, usually approached in two steps: one first tries to construct a constitutive equation that acts as a closure relation to the governing Navier-Stokes (or generally, continuum mechanics) equations at the mesoscopic level. In a second step, these classical field-theory equations are solved, usually numerically. Most commonly, constitutive equations are ad hoc assumptions guided by experimental data. ${ }^{3}$ One of the few exceptions is the rheology of polymer melts, based on the seminal work of Doi and Edwards. ${ }^{4}$

In this paper, we describe first steps towards an approach to simulate, based on an extended lattice-Boltzmann (LB) simulation method, the macroscopic flow of glass-forming fluids in terms of nonlinear constitutive equations that are motivated by a microscopic theory.

In the case of dense glass-forming fluids, shear thinning prevails as the dominant nonlinear mechanism for not too strong strain rates. Progress towards deriving constitutive equations starting from the microscopic equations of motion has been possible because the slow relaxation of

\footnotetext{
a)Electronic mail: simon.papenkort@dlr.de
}

density fluctuations proceeds by generic mechanisms where the precise details of the microscopic interactions, beyond excluded-volume entropic forces, are less important. Specifically for colloidal suspensions, starting from the manybody advection-diffusion equation, an integration-throughtransients (ITT) formalism combined with mode-coupling theory (MCT) ${ }^{5-7}$ has been successful. This approach is in particular aimed at describing the interplay between slow dynamics close to the glass transition and externally imposed flow. In principle, this route yields a constitutive equation that is fully determined by the microscopic interactions of the system. Currently, it remains challenging to treat numerically. Even schematically simplified ITT-MCT models ${ }^{8}$ are difficult to solve beyond the steady state. ${ }^{9}$

To make progress, a simplified model has been proposed that incorporates some of the essential ideas and findings of ITT-MCT. Starting from the Maxwell model of linear viscoelastic fluids, one incorporates the acceleration of the slow structural-relaxation dynamics by shear. ${ }^{10,11}$ This nonlinear generalized Maxwell (nlM) model has been used in systems under homogeneous simple shear to discuss, e.g., qualitative features of the flow curves close to the glass transition ${ }^{12}$ or aspects of creeping flow $^{13}$ and large-amplitude oscillatory shear. ${ }^{14}$ Below we present the extension of this model to arbitrary incompressible flow and incorporate it into the low-Mach-number Navier-Stokes equations to address the pressure-driven Poiseuille flow through channels.

Idealized glass formers are yield-stress fluids, i.e., at the glass transition they are characterized by a nonvanishing stress even in the limit of small strain rates. The signature of a yield stress in Poiseuille flow is the appearance of a non-parabolic velocity profile that is almost flat in the center of the channel, causing the fluid to move as a "plug" in this inner region where the applied force is not able to overcome the yield stress. This has been confirmed in direct 
molecular-dynamics simulations of a glass-forming fluid. ${ }^{15}$ We confirm below that the simple nlM model captures this phenomenology and allows for an analytical solution in steady state. The transient evolution from equilibrium to steady state after application of the driving pressure, and the relaxation back to the quiescent state are studied by numerically solving the corresponding Navier-Stokes equations. A particular feature of the glass forming fluid that is captured naturally by the nlM model is the appearance of large normalstress differences that cause the central plug to be subject to an additional pressure. In channels with constant crosssection, laminar flow continues to be a valid solution. But flow-induced pressure changes may play a decisive role in understanding shear-localization phenomena that are characteristic of many amorphous materials. ${ }^{16}$

The mesoscopic method we employ to solve the Navier-Stokes equations for small velocities is the LB simulation. ${ }^{17-20}$ This method has become increasingly popular over the last decades, because of its conceptual simplicity, and because it is well adapted to parallel computing. On a suitably chosen spatial lattice, one introduces local densities corresponding to discrete velocity vectors. The LB simulation evolves these densities by a sequence of streaming and collision steps. The collision operator relaxes the local distribution towards an equilibrium form, suitably chosen to ensure that in the continuum limit one recovers the Navier-Stokes equations for a Newtonian fluid. The simplest form, the BhatnagarGross-Krook (BGK) collision operator, involves just one relaxation time $\tau_{\mathrm{LB}}$. This is then directly related to the Newtonian shear viscosity.

A simple way of extending the LB method to deal with non-Newtonian fluids, pioneered by Aharonov and Rothman, ${ }^{21}$ is to adjust the BGK relaxation time $\tau_{\mathrm{LB}}(\dot{\gamma})$ locally depending on a (scalar) measure of the local shear rate $\dot{\gamma}$. However, this adjusts just one scalar property, retaining the Newtonian-fluid-like relation between shear and bulk viscosities and neglecting tensorial aspects of the constitutive equation. One should then rather speak of a generalized Newtonian fluid with a locally varying effective viscosity $\eta_{\text {eff }}(\dot{\gamma})$. Nevertheless, the steady-state results have been shown to be rather accurate in simple flow geometries, whenever analytical results are available for comparison. ${ }^{22,23}$ In recent years, a number of studies have employed variants of this generalizedNewtonian LB, addressing also more complicated geometries and the flow through porous media, see, e.g., Refs. 24-30, or the extension to an implicit scheme regarding the calculation of the collision rate. ${ }^{31}$ (A recent more comprehensive overview of a growing body of work is found in Ref. 32.) The generalization to an LB scheme on unstructured grids proposed by Succi and co-workers also uses this description of a generalized Newtonian fluid. ${ }^{33}$ The temporal evolution of the local relaxation time can be implemented by a finite-difference scheme in order to capture time-dependent thixotropic rheology. ${ }^{34,35}$

To incorporate truly non-Newtonian fluids into LB, addressing their tensorial character, one can adjust the LB equilibrium distribution function in a suitable manner. Such algorithms are closely related to so-called lattice kinetic schemes. ${ }^{36-38}$ One may also exploit that the gradient of the
non-Newtonian stresses appears equivalently to an external force density in the Navier-Stokes equations. The dynamics of the non-Newtonian forces is then traced either fully within the LB scheme at the cost of introducing an enlarged set of lattice-node densities, ${ }^{39-41}$ or through suitable finitedifference solvers that are coupled to the LB time evolution, leading to so-called hybrid-LB schemes. ${ }^{42-44}$ Recent, quite different, approaches worth mentioning are to model complex-fluid phenomena through extended LB, where either multiple lattice-density species are introduced and/or the collision rules are adjusted to achieve the desired result. ${ }^{45-47}$ The idea is, opposed to simply view the LB technique as an efficient tool to solve the standard Navier-Stokes equations, to explore the freedom of constructing Boltzmann-equationbased models (which LB adeptly solves), and incorporate the desired physical mechanisms already on the level of the collision term. This provides a promising route, e.g., also in modeling of turbulent flow. ${ }^{48}$

In the following, we present a modified LB scheme in the spirit of hybrid-LB methods that allows to naturally incorporate non-Newtonian stresses, including flow-induced pressure and normal-stress differences relevant close to the glass transition. While our scheme can be extended to a fully fledged hybrid-LB method coupled to a constitutive-equation solver, we focus here on a constitutive equation that is local in time for simplicity. The method is outlined in Sec. II. We then describe the constitutive equation based on the nonlinear generalized Maxwell model in Sec. III, together with some analytical results used to check the accuracy of the simulation. We then present the results for pressure-driven 2D channel flow in Sec. IV.

\section{LATTICE BOLTZMANN SIMULATIONS}

\section{A. Method for non-Newtonian fluids}

We consider a fluid of local mass density $\rho(\vec{r}, t)$ and velocity $\vec{u}(\vec{r}, t)$, whose evolution is described by the Navier-Stokes equations for the momentum density $\vec{J}(\vec{r}, t)$ $=\rho(\vec{r}, t) \vec{u}(\vec{r}, t)$,

$$
\begin{gathered}
\partial_{t} \rho+\vec{\partial} \cdot \vec{\jmath}=0, \\
\partial_{t} j_{\alpha}+\partial_{\beta}\left(\rho u_{\alpha} u_{\beta}\right)=-\partial_{\alpha} p_{0}+\partial_{\beta} \Sigma_{\alpha \beta}+f_{\alpha}^{\mathrm{ex}} .
\end{gathered}
$$

Greek indices denote the Cartesian components of the fields, and summation over repeated indices is implied. In the momentum flux, there appears the Eulerian advection term $\rho \vec{u} \vec{u}$, possibly an externally imposed body-force density $\vec{f}^{\mathrm{ex}}$, and a surface term encoded in the stress tensor $\boldsymbol{\Pi}=p_{0} \mathbf{1}-\boldsymbol{\Sigma}$. The thermodynamic pressure $p_{0}$ is assumed to depend on the conserved quantities only; it is given by an equation of state. Non-uniform flow gives rise to stress terms that depend on the velocity gradients, $\boldsymbol{\Sigma}=-\delta p \mathbf{1}+\overline{\mathbf{\Sigma}}$; these are split into an isotropic "nonequilibrium pressure" $\delta p=-(1 / d) \operatorname{tr} \Sigma$, where $d$ is the spatial dimension, and a traceless deviatoric part $\overline{\boldsymbol{\Sigma}}$. (We use an overbar to denote the traceless part of any tensor.) These quantities need to be supplied by a rheological constitutive equation. 
Newtonian fluids obey the simplest admissible linear constitutive equation, $\boldsymbol{\Sigma}^{\mathrm{N}}=\eta \overline{\boldsymbol{D}}+\eta_{b} \mathbf{1} \operatorname{tr} \boldsymbol{\kappa}$, where $\kappa_{\alpha \beta}=$ $\partial_{\beta} u_{\alpha}$ is the velocity gradient tensor, and $\boldsymbol{D}=\boldsymbol{\kappa}+\boldsymbol{\kappa}^{T}$ its symmetrized form. In the following, it will be useful to separate a Newtonian contribution from the overall stress tensor, identifying the non-Newtonian stresses, $\boldsymbol{\Sigma}=\boldsymbol{\Sigma}^{\mathrm{N}}+\boldsymbol{\Sigma}^{\mathrm{nN}}$.

For nearly-constant-density incompressible flow, $\vec{\partial} \cdot \vec{u}$ $=0$, and the equation of state can be reduced to its firstorder density variation, $p_{0}(\rho) \approx p_{0}\left(\rho_{0}\right)+c_{s}^{2}\left(\rho-\rho_{0}\right)$, where $\rho_{0}$ is some reference density, and $c_{s}$ is the speed of sound. The lattice Boltzmann simulation approximates the NavierStokes equations at low Mach numbers, $M a \sim u / c_{s} \ll 1$, by allowing for weak compressibility. In treating non-Newtonian fluids, care has to be taken since the nonequilibrium pressure may be nonzero even in incompressible flow. Due to the equation of state, this translates to a flow-induced density variation that is not present for Newtonian incompressible fluids. Below we introduce a method designed to take care of this additional density variation. The justification in terms of a ChapmanEnskog expansion is given in the Appendix.

Consider a uniform spatial grid with lattice spacing $\delta x$. The LB scheme evolves a set of lattice density distributions $n_{i}$ by a discrete collision-and-streaming update,

$$
\begin{aligned}
n_{i}\left(\vec{r}+\vec{c}_{i} \delta t, t+\delta t\right) & =n_{i}^{*}(\vec{r}, t) \\
& =n_{i}(\vec{r}, t)+\Delta_{i}[n(\vec{r}, t)]+F_{i},
\end{aligned}
$$

where $\delta t$ is the time step of the simulation and the $\vec{c}_{i}$ are a set of discrete velocities, suitably chosen to ensure the desired continuum limit. The $n_{i}^{*}$ are referred to as the post-collision distributions. $\Delta_{i}$ is the collision operator, constructed to restore the Newtonian-fluid case in the continuum limit. $F_{i}$ is a driving term and can be constructed to account for the external body-force density, although this is a nontrivial matter. ${ }^{49,50}$ We will use it in addition to include non-Newtonian stresses.

The collision operator $\Delta_{i}$ relaxes the lattice densities to a lattice-equilibrium distribution $n_{i}^{\mathrm{eq}}$. The latter is chosen to depend only on the equilibrium properties of the fluid, i.e., its density $\rho$ and velocities $\vec{u}$. Let us define

$$
\rho=\sum_{i} n_{i}^{\mathrm{eq}}, \quad \vec{J}=\sum_{i} \vec{c}_{i} n_{i}^{\mathrm{eq}} .
$$

This reduces to the standard choice in previous LB algorithms. However, one often encounters the simplification to replace the $n_{i}^{\text {eq }}$ (that are still to be specified) with the current populations $n_{i}$. This simplification is no longer justified in the presence of a forcing term $F_{i}$, as has been discussed in detail for the case where an external body force is modeled. ${ }^{51}$

A second-order accurate expression for the equilibrium distribution is

$$
n_{i}^{\mathrm{eq}}(\rho, \vec{u})=a^{c_{i}} \rho\left(1+\frac{\vec{u} \cdot \vec{c}_{i}}{c_{s}^{2}}+\frac{\left(c_{i \alpha} c_{i \beta}-c_{s}^{2} \delta_{\alpha \beta}\right) u_{i \alpha} u_{i \beta}}{2 c_{s}^{4}}\right),
$$

where the set of velocities $\vec{c}_{i}$ obeys the symmetries of a cubic lattice. For our 2D calculations, the simplest choice is the so-called D2Q9 grid with lattice velocities $\vec{c}_{0}=(0,0)$, $\vec{c}_{1 \ldots 4} / c=( \pm 1,0),(0, \pm 1)$, and $\vec{c}_{5 \ldots 8} / c=( \pm 1, \pm 1)$. Here, $c$ $=(\delta x) /(\delta t)$ is the lattice-velocity unit. The weights $a^{c_{i}}$ depend only on the magnitude of the velocity vector and are, for the
D2Q9 model, $a^{0}=4 / 9, a^{1}=1 / 9$, and $a^{\sqrt{2}}=1 / 36$. The model results in a speed of sound $c_{s}=c / \sqrt{3}$, and the equation of state is the linearized expression for small density variations, $p_{0}=\rho c_{s}^{2}$; recall that a constant pressure offset is irrelevant in incompressible flow.

The simplest choice for the collision operator is the single-relaxation-time BGK model, where

$$
\Delta_{i}=-\frac{1}{\tau_{\mathrm{LB}}}\left(n_{i}-n_{i}^{\mathrm{eq}}\right) .
$$

With this choice, the LB algorithm recovers a Newtonian viscosity in the absence of $F_{i}$,

$$
\eta^{\mathrm{N}}=(\delta t) \rho c_{s}^{2}\left(\tau_{\mathrm{LB}}-1 / 2\right) .
$$

To incorporate the non-Newtonian part of the stress tensor, $\boldsymbol{\Sigma}^{\mathrm{nN}}$, we construct the forcing term according to

$$
\begin{aligned}
F_{i}= & a^{c_{i}}\left\{\frac{-1}{2 c_{s}^{4} \tau_{\mathrm{LB}}} \bar{\Sigma}_{\alpha \beta}^{\mathrm{nN}}\left(c_{i \alpha} c_{i \beta}-c_{s}^{2} \delta_{\alpha \beta}\right)\right. \\
& +\left(1-\frac{1}{2 \tau_{\mathrm{LB}}}\right)\left[(\delta t)\left(\partial_{t} \delta \rho\right)+\frac{f_{\alpha}^{\mathrm{ex}} c_{i \alpha}}{c_{s}^{2}}+\frac{c_{i \alpha} c_{i \beta}-c_{s}^{2} \delta_{\alpha \beta}}{2 c_{s}^{4}}\right. \\
& \left.\left.\times\left(-(\delta t)\left(\partial_{t} \delta \rho\right) u_{\alpha} u_{\beta}+\left(u_{\beta} f_{\alpha}^{\mathrm{ex}}+u_{\alpha} f_{\beta}^{\mathrm{ex}}\right)\right)\right]\right\}
\end{aligned}
$$

This expression also accounts for a possible external body force $\vec{f}^{\text {ex }}$. The non-Newtonian stresses are split into their traceless part, $\overline{\boldsymbol{\Sigma}}^{\mathrm{nN}}$ and their trace, $\delta p^{\mathrm{nN}}$ related to $\delta \rho$ as detailed below. As a central idea of our derivation, the former only modify the second velocity-moment of the $F_{i}$, much like the Newtonian stresses in the original LB hinge on the second moments of the $n_{i}$ (see the Appendix).

The trace of the non-Newtonian stresses corresponds to a nonequilibrium pressure contribution. In incompressible flow, this is tantamount to a variation in density, $\delta \rho \propto-\delta p^{\mathrm{nN}}$. This term is treated explicitly in Eq. (8), as a simple way to incorporate the advection of flow-induced pressure. For the simulations of channel flow, this term,

$$
\partial_{t} \delta \rho(t)=-\frac{1}{c_{s}^{2} d \delta t}\left(\Sigma_{\gamma \gamma}^{\mathrm{nN}}(t)-\Sigma_{\gamma \gamma}^{\mathrm{nN}}(t-\delta t)\right),
$$

allows the fluid to build up a pressure gradient countering the normal stresses that act from the sheared boundary regions on the central plug, as we will discuss in detail below. If we did not take care of the non-Newtonian pressure contributions via Eq. (9), the effect of normal stresses would be lost.

\section{B. Boundary conditions}

To maintain a constant pressure gradient along a channel of length $L$, we employ generalized periodic boundary conditions (GPBC) proposed by Kim and Pitsch. ${ }^{52}$ One exploits the simple equation of state of the LB scheme and identifies the average density of the outgoing lattice populations at the lowpressure end of the channel with that of the incoming ones at the high-pressure end, suitably scaled. Specifically, denote by $p_{ \pm}$the inlet and outlet pressures, and by $\bar{\rho}_{\text {in/out }}$ the average values of the lattice densities at the inlet and outlet columns, 
averaged over the transverse spatial directions. One then sets

$$
\left.n_{i}^{*}(\rho, \vec{\jmath})\right|_{\text {in }}=n_{i}^{\text {eq }}\left(c_{s}^{-2} p_{+}+\rho_{\text {out }}-\bar{\rho}_{\text {out }}, \vec{J}\right)+\left.n_{i}^{\text {neq* }}\right|_{\text {out }}
$$

for each inlet node and direction $\vec{c}_{i}$ connecting to the corresponding outlet node, and vice versa. The construction copies over any density fluctuations in the transverse directions, only adjusting the average densities.

Keeping the inlet and outlet pressures fixed negates the effect of implementing the non-Newtonian pressure $\delta \rho c_{s}^{2}$. Therefore, when using GPBC, it proves sufficient to construct a body-force term with vanishing zeroth and first moment, keeping the first line only of Eq. (8). Note that because the $F_{i}$ takes the traceless stress tensor, still a non-trivial pressure gradient in transversal flow direction emerges. In steady flows, this is easy to see, as Eq. (9) vanishes and both, the full and the shortened body-force terms, share the same long time limit. For the cases we consider below, the two methods (GPBC without density correction, or body force with correction) yield identical results up to a reinterpretation of the LB density.

The GPBC can be extended to non-steady flows. In the case of switch-on, we pre-initialize the LB densities to give a linear pressure gradient and thereby avoid shock waves which would be accompanied by unwelcome high Mach numbers.

The simulated channel is bounded by hard walls in the transverse direction(s). For their treatment, we employ simple bounce-back boundary conditions. ${ }^{17}$ In some cases, we also compare with velocity-driven planar Couette flow, where the boundary condition at the moving wall was implemented following Zou and He. ${ }^{53}$ These boundary conditions satisfy no-flux conditions into the hard wall as was derived for the original, Newtonian LB algorithm. Strictly speaking, the implementation of boundary conditions for the modified LB scheme needs to be checked again; we leave this for future work. The results shown below imply that our choice of boundary rules is sufficiently accurate.

\section{Numerical details}

In the following calculations, we adjust $\tau_{\mathrm{LB}}=0.9$, which is close to the optimum reducing the error in the shear stress of a Newtonian fluid. ${ }^{54-56}$ We use a grid of $200 \times 20$ lattice nodes for $\theta \leq 100$, where $\theta$ is the ratio of the low-shear viscosity to the high-shear Newtonian viscosity. Higher $\theta$ requires to resolve larger viscosities and viscosity differences, so that we increase the resolution to $400 \times 20$ nodes for $\theta$ $=1000$. The scheme was implemented in the open-source lattice Boltzmann code Palabos. ${ }^{57}$

To evaluate the constitutive equation, we will typically need to evaluate the velocity-gradient tensor $\kappa(t)=(\vec{\partial} \vec{u}(t))^{T}$. It has in general to be evaluated using a finite-difference scheme on the LB lattice. In case the constitutive equation can fully be specified in terms of the symmetric velocity gradients, $\boldsymbol{D}(t)=\boldsymbol{\kappa}(t)+\boldsymbol{\kappa}^{T}(t)$, one can make use of Eq. (A6) and evaluate $\boldsymbol{D}(t)$ directly from the nonequilibrium distributions on a single lattice node. This method of evaluating the symmetric velocity gradients is second-order accurate, ${ }^{23}$ while a simple finite-difference scheme is only accurate to first order. ${ }^{22}$ Note however, that Eq. (A6) is an implicit equation for $\boldsymbol{D}$ that would generally have to be solved by iteration, since the velocity gradient tensor enters nonlinearly on both sides. Furthermore, we will consider a constitutive equation that does not depend on $\boldsymbol{D}(t)$ only, but also on different (coordinate-frame invariant) combinations of $\boldsymbol{\kappa}(t)$. We therefore use second-order accurate three-point finite differences to evaluate the shear-rate tensor.

Let us briefly discuss the relation to previous approaches in modeling non-Newtonian fluids with LB. The most common approach is to adapt $\tau_{\mathrm{LB}}(\dot{\gamma})$ according to Eq. (7), where the Newtonian viscosity is replaced with a given $\eta_{\text {eff }}(\dot{\gamma})$, and to set $F_{i}=0$. The fluid then remains locally Newtonian. As a consequence, tensorial aspects such as the normal stress differences are not taken into account in this class of generalized Newtonian fluids. Furthermore, the LB method becomes unstable if $\tau_{\mathrm{LB}}$ drops to $1 / 2$, and it works best if the relaxation parameter is chosen within some bounds close to unity. Constitutive equations that lead to strong deviations from these bounds are potentially problematic in this approach.

Ignoring the fact that it gives additional information on normal stresses, the method proposed here is of similar computational efficiency as the scheme based on iterating $\tau_{\mathrm{LB}}(\dot{\gamma})$. For a test case with $\theta=10$ and a grid of $200 \times 20$ points, we found both to be 3 to 4 times slower than the original, purely Newtonian LB. On a current single-CPU machine (Intel Corei5 utilizing 1 core) this translates to approximately $5 \times 10^{6}$ lattice-node updates per second. A large part of the computational overhead compared to the Newtonian case goes into the non-local calculation of $\kappa(t)$ via the finite-difference scheme, and into the evaluation of the constitutive equation itself.

Other methods, close relatives of lattice kinetic schemes, modify the LB-equilibrium distribution function $n_{i}^{\mathrm{eq}}$. In its second moment, a non-Newtonian stress contribution is included. In the steady state, we did not find significant differences to our scheme, which we believe to be easier to justify in the non-steady case. There are applications of LB to complex fluids where the route using a forcing term $F_{i}$ is empirically found to be more robust compared to the modification of the distribution function. ${ }^{40}$

A number of schemes exploit the equivalence of $\vec{\partial} \cdot \boldsymbol{\Sigma}$ with an external force density in Eq. (2). This seems to be particularly useful if the scheme to solve the constitutive equation entails evaluation of the stress gradients. For the present case of the constitutive equation introduced below, we find our approach outlined above to be somewhat simpler, but we do not expect significant differences.

\section{NONLINEAR MAXWELL MODEL}

\section{A. Constitutive equations}

To arrive at a constitutive equation for the flow, we start from a generalized Green-Kubo relation for the nonlinearresponse shear stress that has been worked out in the ITT formalism. Note that the original microscopic derivation of ITTMCT was performed for a colloidal suspension. Our use of the Navier-Stokes equation as the macroscopic flow equation is then justified for low $M a$, i.e., when microscopic inertia effects can be neglected. Furthermore, except for the addition of a high-shear Newtonian background viscosity, the model we 
introduce below focuses on slow structural relaxation effects, whose rheological signature is quite generic (and independent on the type of microscopic equations of motion to a large extent; see also Ref. 58).

We assume some general time-dependent incompressible flow, described by the velocity-gradient tensor $\kappa(t)=(\vec{\partial} \vec{u})^{T}$; incompressibility implies $\operatorname{tr} \kappa=0$. We also neglect the advection of stress gradients. If the flow is switched on at $t=0$ in an equilibrated quiescent system,

$$
\boldsymbol{\Sigma}(t)=\int_{0}^{t}\left[-\partial_{t^{\prime}} \boldsymbol{B}\left(t, t^{\prime}\right)\right] G\left(t, t^{\prime},[\kappa]\right) d t^{\prime},
$$

where $\boldsymbol{B}\left(t, t^{\prime}\right)=\boldsymbol{E}\left(t, t^{\prime}\right) \boldsymbol{E}^{T}\left(t, t^{\prime}\right)$ is the Finger tensor, given by the deformation tensor $\boldsymbol{E}\left(t, t^{\prime}\right)$ that describes the transformation of a material vector $\vec{r}^{\prime}$ at an earlier time $t^{\prime}$ to a vector $\vec{r}$ at some later time $t$. The deformation tensor obeys $\partial_{t} \boldsymbol{E}\left(t, t^{\prime}\right)=\boldsymbol{\kappa}(t) \boldsymbol{E}\left(t, t^{\prime}\right)$ and $\partial_{t^{\prime}} \boldsymbol{E}\left(t, t^{\prime}\right)=-\boldsymbol{E}\left(t, t^{\prime}\right) \boldsymbol{\kappa}\left(t^{\prime}\right)$, with initial condition $\boldsymbol{E}(t, t)=\mathbf{1}$.

The function $G\left(t, t^{\prime},[\kappa]\right)$ is a generalized dynamical shear modulus, given microscopically as a stress-stress autocorrelation function. It will in general depend on two time arguments $t$ and $t^{\prime}$ separately, while in steady state this dependence reduces to one on $t-t^{\prime}$ only. The third argument indicates a dependence on the full flow history at all previous times $t^{\prime} \leq t$. In linear response, this dependence can be neglected, but it is essential to describe non-Newtonian fluids. The principle of material objectivity suggests that the flow history enters the dynamical shear modulus only through the invariants of the Finger tensor (as in the schematic model of Ref. 8), or through invariants of the symmetrized shear-rate tensor $\boldsymbol{D}$ (the simplified case considered below).

In quiescent dense liquids, $G\left(t, t^{\prime}\right)$ typically decays on a slow structural relaxation time scale $\tau$ much larger than the microscopic time scale $\tau_{0}$. Since $\tau \gg \tau_{0}$, visco-elastic effects arise in a large intermediate time window. We consider flows of some characteristic rate $\dot{\gamma}$, where the dressed Péclet number $P e=\dot{\gamma} \tau \gg 1$, but the bare $P e_{0}=\dot{\gamma} \tau_{0} \ll 1$. It is then convenient to model the short-time contributions to the viscosity as quasi-instantaneous, setting

$$
\boldsymbol{\Sigma}(t)=\boldsymbol{\sigma}(t)+\eta_{\infty} \boldsymbol{D}(t) .
$$

Formally, this is achieved by assuming $G\left(t, t^{\prime}\right)=G_{\text {micr }}\left(t, t^{\prime}\right)$ $+G_{\text {struc }}\left(t, t^{\prime}\right)$ to consist of a slowly relaxing structural part $G_{\text {struc }}\left(t, t^{\prime}\right)$ obeying $G_{\text {struc }}(t, t)=G_{\infty}$, and a short-time contribution $G_{\text {micr }}\left(t, t^{\prime}\right) \approx\left(G_{0}-G_{\infty}\right) \Theta\left(\epsilon-\left(t-t^{\prime}\right)\right)$ modeled by a Heaviside function. Inserting into Eq. (11) and taking the limit $\epsilon \rightarrow 0$, we can identify $\left(G_{0}-G_{\infty}\right) \epsilon=\eta_{\infty}=: G_{\infty} \tau_{0}$ as the short-time Newtonian viscosity. In rheological terms, $G_{0}$ corresponds to the high-frequency shear modulus probed at $(t$ $\left.-t^{\prime}\right) \ll \tau_{0}$, while $G_{\infty}$ is the low-frequency Maxwell plateau modulus. Note that $\sigma$ in Eq. (12) is not necessarily traceless.

From the time derivative of Eq. (11), one obtains

$$
\begin{gathered}
\dot{\boldsymbol{\sigma}}(t)-\boldsymbol{\kappa}(t) \cdot \boldsymbol{\sigma}(t)-\boldsymbol{\sigma}(t) \cdot \boldsymbol{\kappa}^{T}(t)=\boldsymbol{D}(t) G_{\infty} \\
+\int_{0}^{t}\left[-\partial_{t^{\prime}} \boldsymbol{B}\left(t, t^{\prime}\right)\right] \partial_{t} G\left(t, t^{\prime},[\boldsymbol{\kappa}]\right) d t^{\prime}
\end{gathered}
$$

dropping the subscript on $G_{\text {struc }}$ for convenience. The terms on the left-hand side are the upper-convected derivative of the stress tensor. ${ }^{59}$

Assume now steady-state flow for a class of generalized Maxwell models (known in the rheology literature as White-Metzner models $\left.{ }^{60}\right)$, where $\partial_{t} G\left(t, t^{\prime},[\kappa]\right)$ $=\left(-1 / \tau_{M}[\kappa]\right) G\left(t-t^{\prime},[\kappa]\right)$ with a relaxation time that is allowed to depend on the deformation rate. The integral on the rhs of the above equation then yields $-\sigma / \tau_{M}$, and using $\dot{\boldsymbol{\sigma}}=\mathbf{0}$ we arrive at the formal steady-state solution

$$
\sigma_{\mathrm{ss}}=\sum_{n \geq 1} G_{\infty} \tau_{M}^{n} \boldsymbol{d}^{(n)},
$$

where we have defined symmetric matrices

$$
\boldsymbol{d}^{(n)}:=\sum_{m=0}^{n}\left(\begin{array}{c}
n \\
m
\end{array}\right) \boldsymbol{\kappa}^{m} \cdot \boldsymbol{\kappa}^{T^{n-m}},
$$

obeying $\boldsymbol{d}^{(0)}=\mathbf{1}, \boldsymbol{d}^{(1)}=\boldsymbol{D}$, and $\boldsymbol{\kappa} \boldsymbol{d}^{(n)}+\boldsymbol{d}^{(n)} \boldsymbol{\kappa}^{T}=\boldsymbol{d}^{(n+1)}$. (Note that in general, $\boldsymbol{d}^{(n)} \neq \boldsymbol{D}^{n}$.) For flows with constant geometry, $\boldsymbol{d}^{(n)}=\left.\partial_{t}^{n} \boldsymbol{B}\left(t, t^{\prime}\right)\right|_{t^{\prime}=t}$. In general, it is nontrivial to ensure that the infinite sum (14a) converges. For simple shear flow, $\kappa_{\alpha \beta}=\dot{\gamma} \delta_{\alpha x} \delta_{\beta y}$, we have $\boldsymbol{\kappa} \cdot \boldsymbol{\kappa}=\mathbf{0}$, so that $\boldsymbol{d}^{(2)}$ $=2 \boldsymbol{\kappa} \cdot \boldsymbol{\kappa}^{T}$ and all terms for $n>2$ vanish. The model then contains a shear stress, $\sigma_{x y}=G_{\infty} \dot{\gamma} \tau_{M}$, and a first normal-stress difference $N_{1}=\sigma_{x x}-\sigma_{y y}=2 G_{\infty} \dot{\gamma}^{2} \tau_{M}^{2}$. The two quantities obey a simple scaling, $G_{\infty} N_{1} / \sigma_{x y}^{2}=2$. Since the other diagonal elements of the stress tensor vanish, we also have $\delta p=-(1 / d) \sigma_{x x}=-(2 / d) G_{\infty} \dot{\gamma}^{2} \tau_{M}^{2}$ (where $d$ denotes the spatial dimension).

Equation (14a) with constant $\tau_{M} \equiv \tau$ defines the upperconvected Maxwell model (UCM) ${ }^{2}$ under steady shear, with a (low-shear) Newtonian viscosity $\eta=G_{\infty} \tau$. For some choices of $\boldsymbol{\kappa}$, Eq. (14a) diverges in the UCM: in planar extensional flow, we have $\kappa_{x x}=-\kappa_{y y}=\dot{\epsilon}$ and all other $\kappa_{\alpha \beta}=0$. Equation (14a) then yields $\sigma_{x x}=2 G_{\infty} \dot{\epsilon} \tau /(1-2 \dot{\epsilon} \tau)$, valid only for $\dot{\epsilon} \tau<1 / 2$. This is a well-known deficiency of the UCM which is cured in the nonlinear model we discuss below.

In shear-thinning fluids, the structural relaxation time $\tau$ interferes with the time scale set by the external perturbation, $1 / \dot{\gamma}$. Correlation functions such as $G(t)$ under strong shear hence decay on a time scale that is the equilibrium relaxation time $\tau$ as long as shear is weak ( $P e \ll 1)$, but shear induced if $P e \gg 1$. In an $a d h o c$ way, this can be modeled in a nonlinear generalized Maxwell (nlM) model by letting

$$
\tau_{M}^{-1}=\tau^{-1}+I I_{D}^{1 / 2} / \gamma_{c}
$$

with some model parameter $\gamma_{c}$. Here, $I I_{D}=(1 / 2) \operatorname{tr} \boldsymbol{D}^{2}$ (for incompressible flow) is the second invariant of the symmetric velocity-gradient tensor and generalizes the notion of a scalar shear rate to non-simple flow geometries. In simple shear, $I I_{D}=\dot{\gamma}^{2}$, while in planar extensional flow, $I I_{D}=(2 \dot{\epsilon})^{2}$. The assumption that the structural-relaxation time $\tau_{M}$ is modified by the instantaneous shear rate goes back to White and Metzner, ${ }^{60}$ who made no explicit statement about the possible functional dependence. The present form, Eq. (15), is a material objective tensorial generalization of the nIM model for simple shear proposed recently as a caricature of the MCT mechanism for shear thinning. ${ }^{10-12}$ According to this 
interpretation, the coefficient $\gamma_{c}$ models a typical strain amplitude relevant for the shear-induced breaking of nearestneighbor cages. Guided by the observation that the yield strain of dense hard-core suspensions is close to $10 \%$, we set $\gamma_{c}$ $=0.1$ in numerical calculations. Setting $\gamma_{c} \ll 1$, the model based on Eq. (15) cures the deficiency of the linear-response UCM model, since the infinite sum in Eq. (14a) can be forced to converge for any given flow-rate tensor. ${ }^{61}$

The nlM model incorporates a dynamic yield stress if $\tau$ $\rightarrow \infty$ : let us introduce $\kappa(t)=K(t) \kappa_{0}$ where the flow rate $K(t)$ can be chosen positive without loss of generality. We assume here that the geometry of the flow does not change over time. Then, $I I_{D}=K(t)^{2} I I_{D_{0}}$ and the constitutive equation, Eq. (14a), for $\tau \rightarrow \infty$ gives $\boldsymbol{\Sigma}=\boldsymbol{\sigma}_{y}+\eta_{\infty} \boldsymbol{D}$ with

$$
\boldsymbol{\sigma}_{y}=\sum_{n \geq 1} G_{\infty}\left(\frac{\gamma_{c}}{I I_{D_{0}}^{1 / 2}}\right)^{n} \sum_{m=0}^{n}\left(\begin{array}{c}
n \\
m
\end{array}\right) \boldsymbol{\kappa}_{0}^{m} \boldsymbol{\kappa}_{0}^{T^{n-m}}
$$

Our constitutive equation can hence be classified in rheological terms as that of a Bingham fluid in the limit $\tau \rightarrow \infty$.

Beyond the steady state, one cannot expect Eq. (13) to reduce to a differential equation. While one could consider $\partial_{t} G\left(t, t^{\prime}\right)=\left(-1 / \tau_{M}(t)\right) G\left(t, t^{\prime}\right)$ together with Eq. (15) as a time-dependent generalization of the model, this would imply that the dynamical shear modulus depends on the flow only through an accumulated strain $\int_{t^{\prime}}^{t} I I_{D}^{1 / 2}(s) d s$. In cases like large-amplitude oscillatory shear (LAOS), this does not appear to be plausible, since it implies that memory effects "come back" even in the plastic-flow regime after a cycle of strong shear. It can be argued that in dense liquids under strong flow, the instantaneous rate $I I_{D}^{1 / 2}$ itself should control a decay rate. In a class of constitutive equations known as fluidity models, ${ }^{62-64}$ one essentially imposes $\tau_{M}(t)$ to obey another differential equation. Another possibility is to consider a flow dependence of $\tau_{M}$ that is not instantaneous in the time $t$, but influenced by earlier times $t^{\prime} .{ }^{13,14}$ This results in an integral generalized-Maxwell models that no longer allow the reduction of Eq. (11) to a differential constitutive equation.

Such integral constitutive equations can be treated with the algorithm outlined above, ${ }^{65}$ at the cost of much higher computational effort. In the present paper, we will restrict ourselves to the generalized Maxwell model defined by Eqs. [(14a) and (14b)] and (15), where $\kappa$ and hence $\tau_{M}$ are replaced by their time-dependent, instantaneous values. This instantaneous nonlinear Maxwell (inlM) model amounts to focussing on the physics of a yield-stress fluid, and neglects effects of visco-elasticity.

Let us also mention that the model emphasizes the temporal history effects over spatial nonlocalities, quite in the spirit of ITT-MCT. In general, one expects Eq. (11) to depend on stress- and strain-rate gradients if the flow field $\kappa(\vec{r}, t)$ is inhomogeneous. This leads to advective contributions of the form $(\vec{v} \cdot \vec{\partial}) \sigma$, and possibly further gradient terms entering the definition of the dynamical shear modulus $G\left(t, t^{\prime}\right)$. Our inlM model neglects these effects, which is certainly reasonable for laminar flow along the channel.

\section{B. Channel flow}

The stationary laminar velocity profile in a pressure driven 2D channel flow of a non-Newtonian fluid described by the nonlinear Maxwell model introduced above can be calculated analytically. This solution will serve as a useful reference case to check the LB scheme. Consider a channel of width $2 H$ (taken in the $y$-direction) and a pressure drop $\Delta p$ per unit length ( $x$-direction). We introduce dimensionless quantities by $s=t / \tau_{0}$ and $d=y / H$; the streaming velocity of interest then is $v=u_{x} \tau_{0} / h$. We assume spatial homogeneity along the flow direction. In incompressible flow, $u_{y}$ then has to vanish identically, and the Navier-Stokes equation combined with the inlM model reduces to

$$
R e \partial_{s} v=\wp H+\partial_{d}\left[\left(1+\frac{\theta}{1+\theta\left|\partial_{d} v\right| / \gamma_{c}}\right) \partial_{d} v\right]
$$

with $\wp=\Delta p / G_{\infty}$. Re $=\rho H^{2} /\left(\eta_{\infty} \tau_{0}\right)$ is the (worst-case) Reynolds number of the problem. The parameter $\theta=\tau / \tau_{0}$ quantifies the relative enhancement of the low-shear viscosity over the Newtonian high-shear one; $\theta=0$ corresponds to a Newtonian fluid. As the glass transition is approached, $\theta \rightarrow \infty$.

We further assume no-slip boundary conditions imposed at $d= \pm 1$. Symmetry dictates $\partial_{d} v=0$ for $d=0$, and we anticipate that the velocity gradient does not change sign in either half of the channel. Under these conditions, the flow in the upper half of the channel, in steady state, is given by a quadratic equation for $\partial_{d} v$,

$$
0=\wp H d+\left(1+\theta-\wp H d \theta / \gamma_{c}\right) \partial_{d} v-\left(\theta / \gamma_{c}\right)\left(\partial_{d} v\right)^{2},
$$

which is solved by

$$
\begin{aligned}
\partial_{d} v(d)= & -\frac{\wp H d}{2}+\frac{\gamma_{c}(1+\theta)}{2 \theta} \\
& -\sqrt{\left(\frac{\wp H d}{2}-\frac{\gamma_{c}(1+\theta)}{2 \theta}\right)^{2}+\frac{\wp H d \gamma_{c}}{\theta} .}
\end{aligned}
$$

To obtain the dimensionless velocity $v(d)$, this expression is to be integrated on $d \in[0,1]$ and shifted so that $v(1)=0$ is obeyed.

For small $\theta$, the above expression reduces to $\partial_{d} v(d)$ $=-\wp H d(1-\theta)+O\left(\theta^{2}\right)$, so that the Newtonian parabolic velocity profile expected in Poiseuille flow is recovered. Newtonian profiles are in general expected (for finite $\theta$ ) if the pressure gradient is small, $\partial_{d} v(d)=-\wp H d /(1+\theta)+O\left(\wp^{2}\right)$, or large enough to probe the Newtonian high-shear viscosity, $\quad \partial_{d} v(d)=-\wp H d+\gamma_{c}+O(1 / \wp) \sim-\wp H d$ as $\wp$ $\gg \gamma_{c} /(H d)$.

For $\theta \rightarrow \infty$, a singular boundary layer emerges in Eq. (17), so that one has to distinguish an inner (centerchannel) and outer solution in Eq. (19). For $d>\gamma_{c} /(\wp H)$, we get $\partial_{d} v=-\wp H d+\gamma_{c}+\mathcal{O}(1 / \theta)$, a shifted parabolic profile. For $d<\gamma_{c} /(\wp H)$, a "plug" solution $\partial_{d} v=\mathcal{O}(1 / \theta)$ appears. Specifically,

$$
\partial_{d} v(d) \sim\left\{\begin{array}{ll}
-\wp H d+\gamma_{c} & d>d_{c}=\gamma_{c} /(\wp H) \\
\wp H d \gamma_{c} /\left(p H d-\gamma_{c}\right) / \theta & d<d_{c}
\end{array} .\right.
$$


The expansion in $1 / \theta$ is nonanalytic for $d=d_{c}$, where the two solutions merge. In particular, we do not find a solution for $\theta=\infty$, since the model then predicts $\dot{\gamma}=0$ and a constant shear stress inside the plug, in violation of the Navier-Stokes equation $\sigma_{x y}=-\Delta p H d$. The plug boundary is, in line with physical expectation, simply given by the point where this stress matches the yield stress under shear, $\sigma_{x y}\left(d_{c}\right)=G_{\infty} \gamma_{c}$ $=\left(\sigma_{y}\right)_{x y}$.

According to Eq. (14a), the above steady-state solution implies a normal-stress difference $N_{1}=\sigma_{x x}-\sigma_{y y}$ $=2 G_{\infty}\left(\partial_{y} u_{x}\right)^{2} \tau_{M}^{2}$, and a nonequilibrium stress contribution $\delta p=N_{1} / 2$. The solution with $u_{y} \equiv 0$ is indeed consistent with the Navier-Stokes equations. In the limit $\theta \rightarrow \infty$, there results for the normal stress difference

$$
\sigma_{x x}-\sigma_{y y} \sim\left\{\begin{array}{ll}
2 G_{\infty} \gamma_{c}^{2} & d>d_{c} \\
2 G_{\infty}(\wp H d)^{2} & d<d_{c}
\end{array} .\right.
$$

If the pressure gradient drops below a certain yield value, $\Delta p$ $<\Delta p_{c}=2 G_{\infty} \gamma_{c} /(2 H)$, no flow is found across the entire width of the channel.

The emergence of a yield stress has an interesting consequence for the temporal evolution of the flow profile after the driving pressure is removed (or switched to one below the yield value). While a Newtonian fluid's velocity will decay exponentially in time, the one of a yield-stress fluid of WhiteMetzner type (with a relaxation rate that depends on the instantaneous shear rate) will drop to zero at a finite stopping time. ${ }^{66,67}$

\section{RESULTS}

To test the modified LB scheme, we first discuss the steady-state pressure-driven flow through a 2D channel, comparing with the analytical solution provided by Eq. (19). After that, the transient dynamics will be studied; here, no analytical solution is known, but the exact upper bound for a finite-time singularity where the flow strictly vanishes after removal of the driving pressure gradient, provides a stringent test of the modified LB scheme proposed in this paper.

Our constitutive equation becomes Newtonian for both low and high shear rates: there holds $\boldsymbol{\Sigma} \sim \eta_{\infty} \boldsymbol{D}$ for $\|\boldsymbol{D}\|$ large enough, since in this limit $\sigma$ approaches $\sigma_{y}$ which remains bounded. For small $\|\boldsymbol{D}\|$ on the other hand, we can set $\tau_{M}$ $\sim \tau$, and obtain $\boldsymbol{\Sigma}=\left(\eta+\eta_{\infty}\right) \boldsymbol{D}$. Applying our LB scheme, we have to fix $\tau_{\mathrm{LB}}$, and consequently all lattice units, to match a given Newtonian viscosity. Based on the physical picture, it is tempting to choose the high-shear viscosity $\eta_{\infty}$, and hence to identify $\boldsymbol{\Sigma}^{\mathrm{nN}} \equiv \sigma$. However, we found the accuracy of the LB simulation to be greatly enhanced by choosing $\tau_{\mathrm{LB}}$ to match the larger low-shear viscosity of the Maxwell model, identifying $\Sigma^{\mathrm{nN}}=G_{\infty}\left(\tau_{M}-\tau\right) \boldsymbol{D}$ $+\sum_{n \geq 2} 2 G_{\infty} \tau_{M}^{n} \boldsymbol{d}^{n}$. The fact that this contribution becomes negative does not affect the stability of the scheme as long as the physical viscosity is guaranteed to be positive.

Here and in the following we choose a pressure difference $\Delta p=G_{\infty} / 2 H$, i.e., a pressure drop comparable to the elastic modulus of the fluid. This choice is appropriate for soft matter flow and brings out most clearly the non-Newtonian plug-flow effects.

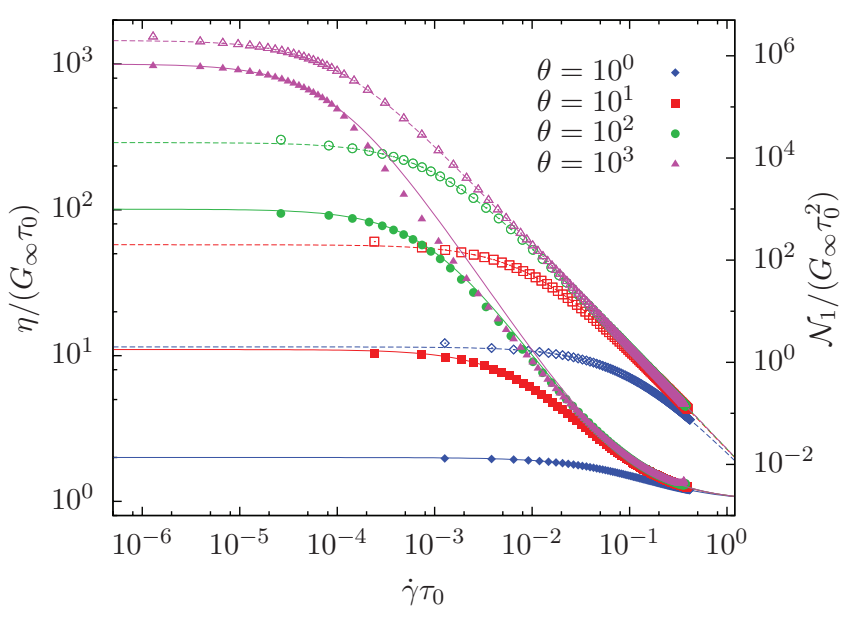

FIG. 1. Shear viscosity $\eta=\sigma_{x y} / \dot{\gamma}$ (filled symbols; left axis) and normal stress coefficient $\mathcal{N}_{1}=\left(\sigma_{x x}-\sigma_{y y}\right) / \dot{\gamma}^{2}$ (open symbols; right axis) as a function of local shear rate $\dot{\gamma}$. Lines are analytical results from the nonlinear Maxwell model for different quiescent relaxation times $\tau=\theta \tau_{0}$, in units of the microscopic relaxation time $\tau_{0}$; symbols are LB simulation results.

\section{A. Stationary profiles}

The steady state properties of the constitutive equation are illustrated in Fig. 1. We show the rate-dependent shear viscosity $\eta(\dot{\gamma})=\sigma_{x y} / \dot{\gamma}$, and the normal stress coefficient $\mathcal{N}_{1}$ $=\left(\sigma_{x x}-\sigma_{y y}\right) / \dot{\gamma}^{2}$, as a function of the local shear rate $\dot{\gamma}=\kappa_{x y}$ in the fully developed channel flow. We will see below that indeed the steady-state solution is to a very good approximation given by $u_{x}(y)$ as the only non-vanishing velocity component, and hence $\kappa_{x y}$ as the only non-zero velocity gradient. Since our constitutive equation is local and does not involve gradient terms, the results shown in Fig. 1 are identical to those obtained in a simple planar shear setup, where a homogeneous shear rate $\dot{\gamma}$ is controlled. This has been checked separately.

The nonlinear Maxwell model describes an increase in both shear viscosity and normal stress coefficient due to slow structural relaxation, parametrized by the large relaxation time $\tau$. The shear viscosity for $\dot{\gamma} \rightarrow 0$ becomes that of a Newtonian fluid (independent on shear rate), and grows as a function of $\tau$. Upon increasing shear rate, as $\dot{\gamma} \tau \approx \gamma_{c}$, shear thinning sets in, because $\tau_{M}$ is no longer controlled by $\tau$, but by $1 / \dot{\gamma}$. As a result, $\eta(\dot{\gamma}) \sim 1 / \dot{\gamma}$, i.e., the model contains the (trivial) shear-thinning exponent -1 . At large $\dot{\gamma}$, the Maxwell contribution to $\eta$ becomes negligible, and a Newtonian fluid obeying $\eta=\eta_{\infty}$ results. This regime is not fully resolved in our channel flow simulations, but is easily accessible to the LB scheme in simple shear.

At low shear rates, the fluid is not truly Newtonian, since large normal stress coefficients arise even in incompressible flow. These scale as $\tau^{2}$ in the limit $\dot{\gamma} \rightarrow 0$. In the shear-thinning regime, the normal stress coefficient obeys $N_{1}$ $\sim 1 / \dot{\gamma}^{2}$ as expected by symmetry - recall that upon reversing the flow direction, the diagonal elements of $\sigma$ do not change sign.

As shown in Fig. 1, our LB scheme (results shown as symbols) is able to trace the analytical solution of the nonlinear Maxwell model (lines) over at least six orders of 


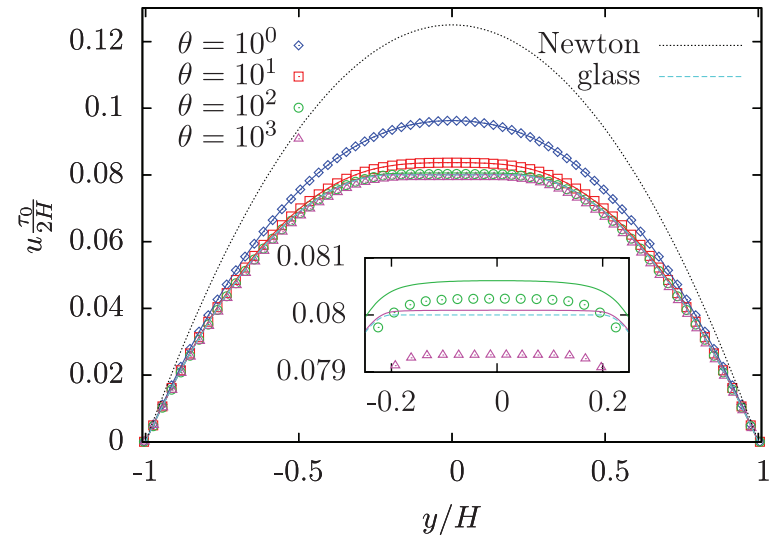

FIG. 2. Steady-state velocity profile in a 2D channel flow driven by a constant pressure gradient $\Delta p /(2 H)=G_{\infty}$, for the nonlinear Maxwell model, for different $\theta=\tau / \tau_{0}$. Symbols are LB results, solid lines are the analytical solutions obtained from Eq. (19). A dashed line (see inset) shows the solution for $\theta \rightarrow \infty$, the dotted line is the parabolic profile for a Newtonian fluid, $\theta=0$.

magnitude in the shear rate, and three orders of magnitude in viscosity variation. (Implying more than six orders of magnitude change in the normal-stress coefficient.) Only at the largest value of $\tau$ considered, $\theta=10^{3}$, some deviations can be seen. We expect that a better lattice resolution will improve these results.

The qualitative features of the flowcurves shown in Fig. 1 are in agreement with many shear-thinning fluids close to a glass transition for small bare Péclet numbers, $P e_{0}=\dot{\gamma} \tau_{0}$ $\ll 1$. They are also in qualitative agreement with calculations based on a schematic MCT model. ${ }^{8}$ At high $P e_{0}$, one usually finds still non-vanishing normal-stress differences, together with an increasing non-equilibrium pressure contribution $\delta p .{ }^{68}$ This is not captured in our model, since we assume a purely Newtonian high-shear viscosity. Augmenting the model to display another Maxwell-type relaxation on the time scale $\tau_{0}$ would be closer to experimental and MD simulation results. In the following, we focus on small shear rates, so that this difference is not relevant here.

We now turn to the velocity profiles of the planar channel flow. Figure 2 compares the velocity profiles obtained by our LB simulation to the analytic solution for the strictly incompressible case, Eq. (19), for various $\theta$. For $\theta \rightarrow 0$, the familiar parabolic Poiseuille flow profile of a Newtonian fluid is recovered (LB results not shown). As $\theta$ increases, the center velocities decrease, while the velocity gradients flatten. As $\theta \rightarrow \infty$, a "plug" of unsheared liquid develops in the center of the channel. For $\theta \leq 10^{3}$, the LB results (symbols in Fig. 2) are in good agreement with the analytic prediction. The largest deviations are seen in the plug for large $\theta$, as shown in the inset of the figure. Even for $\theta=10^{3}$, the relative deviation in the velocity profile is less than $1 \%$. This remaining error is largely governed by the LB grid resolution. This is similar to LB simulations where a "scalar" constitutive equation is employed, either in terms of an extra forcing term $F_{i}$, or through a local adaption of $\tau_{\mathrm{LB}}$ as discussed in the Introduction. Using both these schemes for comparison, we found similar errors as the ones shown in Fig. 2.

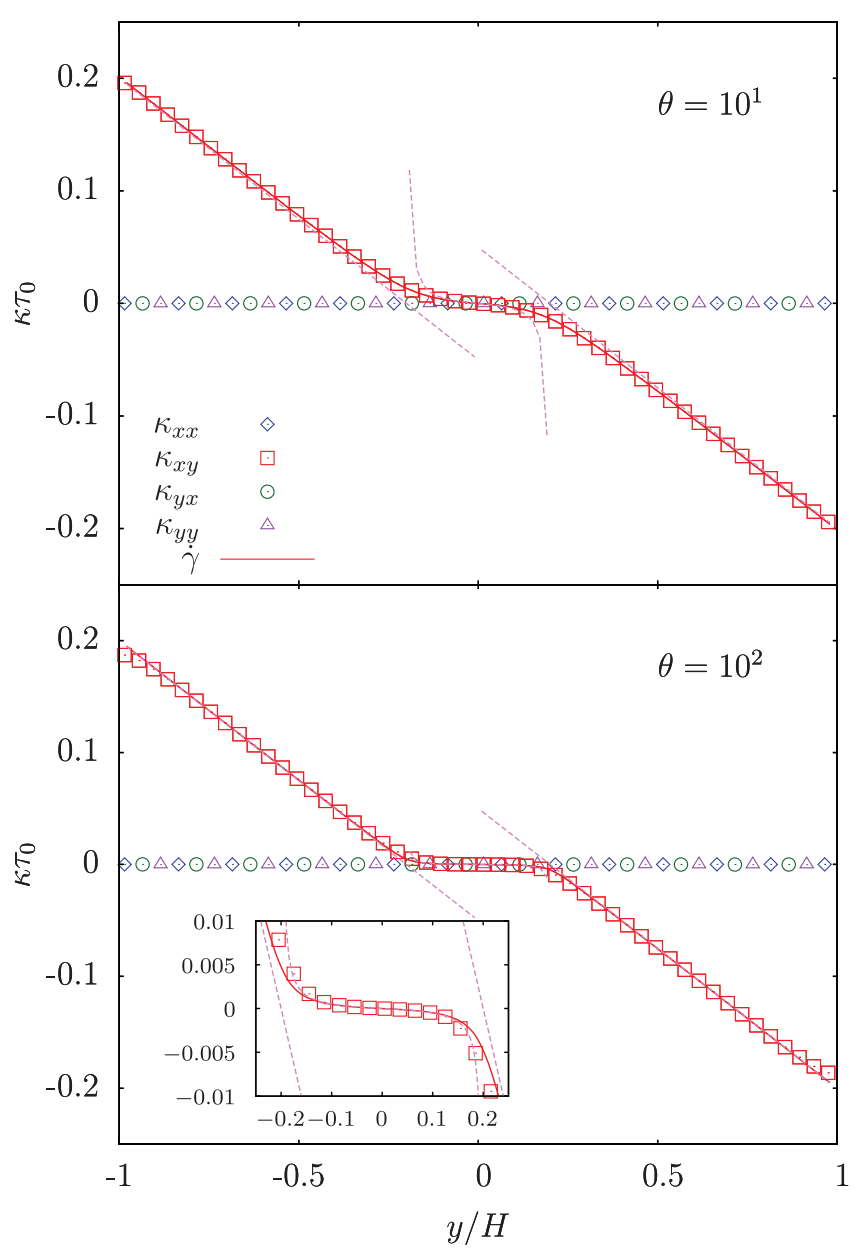

FIG. 3. Velocity gradient tensor $\kappa$ from LB simulations of the nonlinear Maxwell model, in pressure-driven 2D channel flow. The dashed lines show the prediction of the asymptotes Eq. (20), the solid lines the analytic result Eq. (19).

The half-width $w$ of the plug follows from Eq. (20), $w=2 G_{\infty} \gamma_{c} /(\Delta p H)=0.2$, and already $\theta=10^{2}$ is very close to the theoretical $\theta \rightarrow \infty$ solution (shown in Fig. 2 as a dotted line). One can view finite $1 / \theta$ as a regularization parameter as it is often employed in numerical calculations involving Bingham or other yield-stress fluids. ${ }^{69}$ In fact, the case $\theta \rightarrow \infty$ is an idealization that is not achieved in reality, as even in the glass, some residual relaxation processes persist.

The emergence of a plug region is even more clearly seen in the velocity gradients. Figure 3 shows the elements of the velocity-gradient tensor $\boldsymbol{\kappa}$ for the pressure-driven channel flow discussed in connection with Fig. 2, for $\theta=10$ and $\theta=100$. The only element that is numerically different from zero is $\kappa_{x y}$, as expected from the incompressibility condition. With our choice of grid parameters, the largest error occurs near the channel inlet/outlet boundaries, where $\kappa_{x x}$ $=\mathcal{O}\left(5 \times 10^{-8}\right)$. Symbols in Fig. 3 show LB simulation results; they agree very well with the analytical prediction, Eq. (19), shown as solid lines. Furthermore, even for the moderate $\theta$, the asymptotic result for $\theta \rightarrow \infty$, Eq. (20), already describes the velocity gradients surprisingly well (dashed lines).

Figure 4 shows the elements of the stress tensor corresponding to Fig. 2 with $\theta=10$ and $\theta=100$. As shown in the 


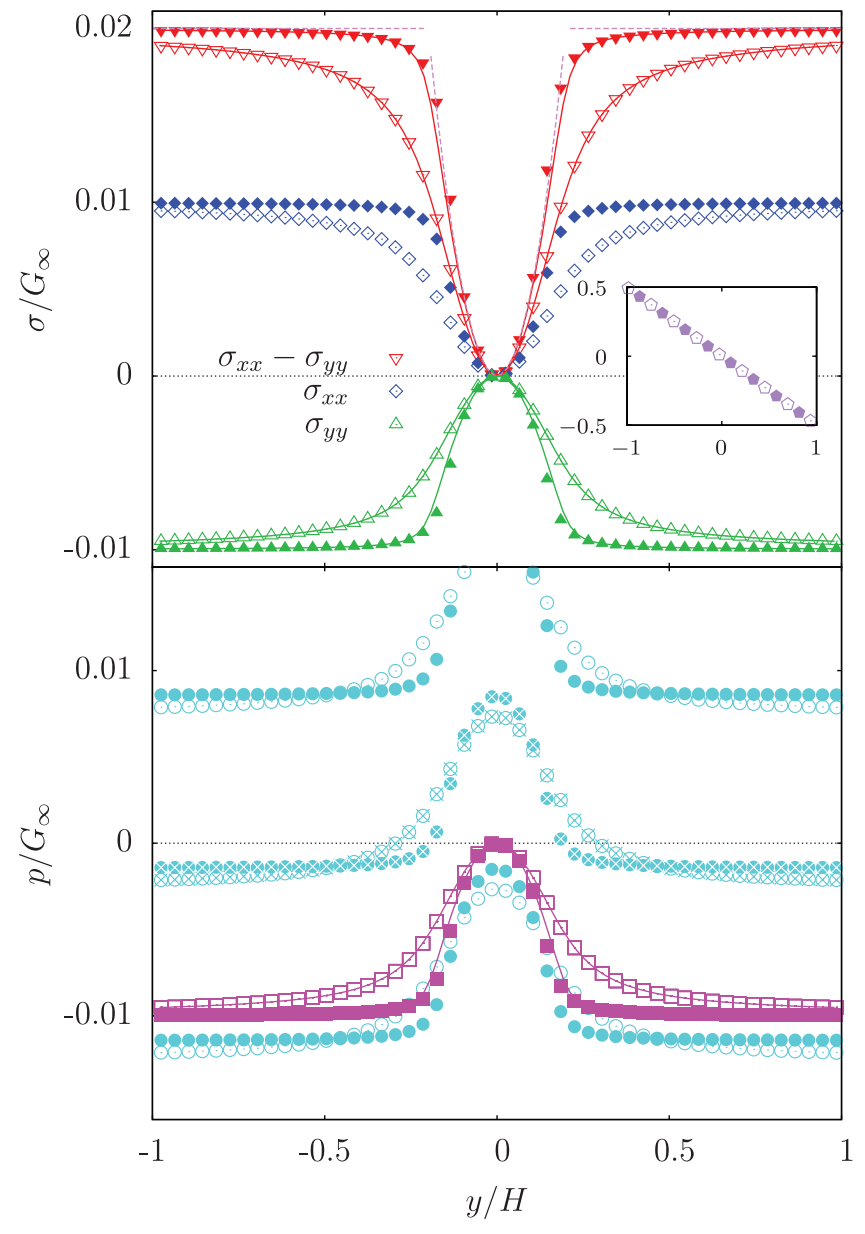

FIG. 4. Stress tensor elements $\sigma_{\alpha \beta}$ for pressure-driven channel flow with $\theta$ $=10$ (open symbols) and $\theta=100$ (filled symbols). Upper panel: Diamonds (triangles) show $\sigma_{x x}\left(\sigma_{y y}\right)$, while inverted triangles are the normal stress difference from the LB simulation. Lines are the analytical calculation from the nonlinear Maxwell model, assuming incompressible flow. The inset shows the expected linear behavior of $\sigma_{x y}$. Lower panel: Circles show different cuts along the channel of the flow-induced pressure when using generalized periodic boundary conditions including a pressure step along the channel. The center-channel position is marked with crosses. If flow is instead driven by a body force, the pressure becomes translation-invariant along the channel (squares).

inset, the shear stress $\sigma_{x y}$ obeys the expected linear behavior dictated by the Navier-Stokes equation. This is the only element of the stress tensor that is nonzero for the "scalar" constitutive equation incorporated in non-Newtonian LB schemes that adjust $\tau_{\mathrm{LB}}$ through iteration.

The normal-stress difference $N_{1}=\sigma_{x x}-\sigma_{y y}$ $=2 G_{\infty} \dot{\gamma}^{2} \tau_{M}(\dot{\gamma})^{2}$ contained in the nonlinear Maxwell model can be evaluated easily from Eq. (19). As demonstrated in Fig. 4, the LB simulation results (circles) are in excellent agreement with this prediction (shown as a solid line) for the values of $\theta$ we investigated. From Eq. (21), we obtain for $\theta$ $\rightarrow \infty$ a constant normal stress difference outside the plug, and a parabolic dependence inside. This is shown in the figure as a dashed line. Already for $\theta=100$, the normal stress coefficient closely follows this asymptotic prediction. In Fig. 4, we also show the individual elements of the deviatoric stress tensor, $\bar{\sigma}_{x x}$ and $\bar{\sigma}_{y y}$, obtained by the LB algorithm. They reconfirm the analytical calculation and highlight the fact that the modified LB algorithm absorbs the isotropic part of the non-Newtonian stresses as an additional pressure.

To elucidate this point, we show in the bottom panel of Fig. 4 the overall pressure as a function of the cross-channel position $y$, for various cuts at constant $x$-position along the channel. The inclusion of pressure effects in the nearly incompressible LB solution is not without subtlety, and we show two possible approaches: cyan symbols in Fig. 4 correspond to simulations with generalized periodic boundary conditions incorporating a fixed pressure difference. Magenta symbols are results obtained with a body force driving the fluid flow; in this case, the overall pressure is translational-invariant along the channel. For all other quantities discussed here, the two methods give results that are numerically indistinguishable; however, for the case of a body force driving the flow, additional care has to be taken to account for the non-Newtonian pressure within LB. The generalized boundary conditions directly control the average pressure and are hence easier to implement in this case. ${ }^{65}$

The appearance of a positive $N_{1}$ causes the fluid to be driven towards the plug, since in the sheared region, forces act perpendicular to the flow direction towards the center and towards the confining walls. The latter forces are balanced by the no-flux boundary conditions. As a result, the pressure inside the plug region increases relative to the one outside.

\section{B. Transient dynamics}

We next consider the transient dynamics when going over from the quiescent state to a flowing steady state, and vice versa, by applying or removing the pressure difference instantaneously.

Figure 5 shows the evolution of the mid-channel velocity profiles after startup of 2D channel flow. In the Newtonian case, an explicit analytical solution is available; ${ }^{70}$ it essentially shows an exponential increase towards the steady-state value. Even for the non-Newtonian case $\theta>0$, no qualitative change is seen. The switch-on solutions are dominated

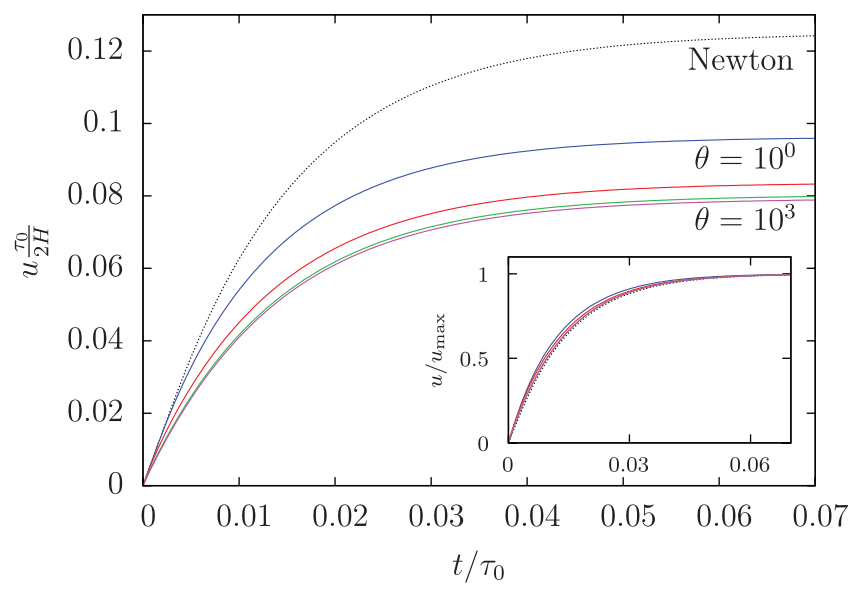

FIG. 5. Evolution of the mid-channel velocity after application of a pressure gradient (startup), for different $\theta$. Nonlinear Maxwell model, LB results. In the inset, the velocities are scaled by the steady state value. 


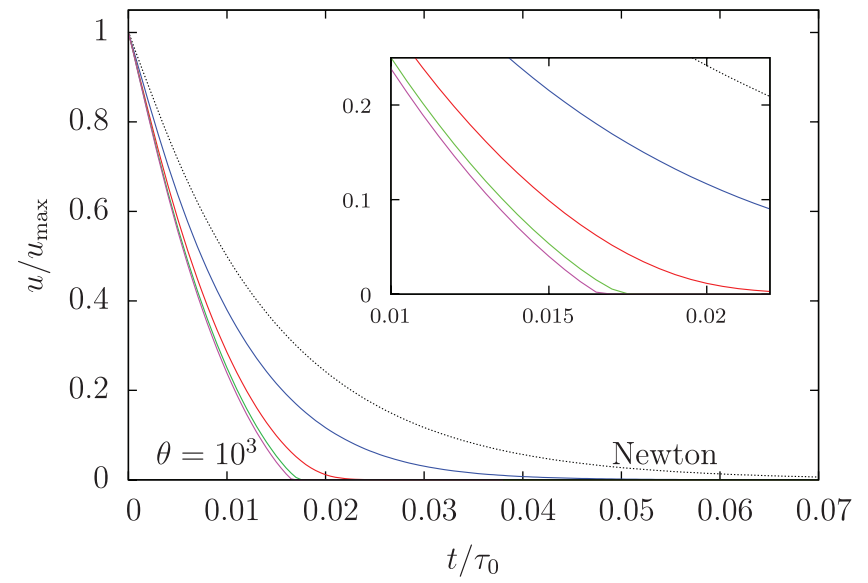

FIG. 6. Evolution of the mid-channel velocity after removal of a constant pressure gradient (cessation). Nonlinear Maxwell model, LB results.

by equating the time-derivative of the velocity with the constant pressure-drop term in Eq. (17), so that the nonlinear contributions from the stress tensor are small. As shown in the inset of Fig. 5, the startup results can almost be scaled on top of each other simply by dividing through the steady-state value.

Figure 6 shows the cessation of pressure-driven channel flow when the applied pressure difference is suddenly removed. Here, the terms that balance in the Navier-Stokes equation are the time derivative and the stress-tensor derivative, so that nonlinear contributions to the latter are much more prominent. For the Newtonian case, start-up and cessation evolution are symmetric in the sense that the corresponding results in Figs. 5 and 6 can be collapsed by a simple linear transformation. ${ }^{70}$ While for any finite $\theta$, the ultimate flow decay is again Newtonian, for large $\theta$ the cessation profiles indicate the stopping-time phenomenon quoted above. As evident from Fig. 6, and consistent with Fig. 2, already $\theta=100$ is representative of the yield-stress fluid case $\theta=\infty$ in this respect.

The LB algorithm is accurate enough to resolve the finitetime singularity within reasonable bounds. We estimate a stopping time of $t \approx 0.016 \tau_{0}$. This is compatible with the upper bound estimated by Huilgol. ${ }^{66}$ Previously, the accuracy of this upper bound was checked in FEM (Finite Element Method) simulations. ${ }^{69,71}$ Our results indicate that the LB algorithm provides similar accuracy for non-Newtonian flows.

Note that the appearance of a finite stopping time is a consequence of the instantaneous nonlinear Maxwell model, or other instantaneous yield-stress constitutive equations, since it is derived from a variational inequality that is local in space and time. Incorporation of viscoelastic effects in the full nonlinear generalized Maxwell model will render the cessation flow phenomenology more complex.

To complete the discussion, Figure 7 shows the temporal evolution of the plug-flow profile after application and after removal of the driving pressure gradient. In startup flow (left panel), the plug-flow profile develops from an initially flat velocity profile, with a central plug whose width gradually decreases until it reaches the steady-state width discussed above. The decay of the velocity profiles after removal of the pressure difference qualitatively follows the inverse sequence of steps.

The asymmetry between startup and cessation of flow induced by the non-Newtonian behavior of the fluid is even more clearly seen in the case of planar Couette flow, where one wall is driven by a fixed velocity that is instantaneously switched on and off. In Fig. 8 we show the resulting buildup and cessation of velocity profiles for this case. Note that in comparison to the pressure-driven channel, we take the Couette-flow channel to be of width $H=L$; this accounts for the mirror symmetry present in the former, but absent in the latter case.

The startup curves shown in Fig. 8 (left panel) are qualitatively identical to those of a Newtonian fluid. Note that in the stationary case, no plug flow develops, since in homogeneous Couette flow the boundary conditions impose a constant shear rate everywhere. On the other hand, the cessation curves (right panel) again develop an intermediate plug, starting from the previously moving wall. They reconfirm qualitatively the results for a different yield-stress fluid model evaluated within finite-element simulations. ${ }^{69}$

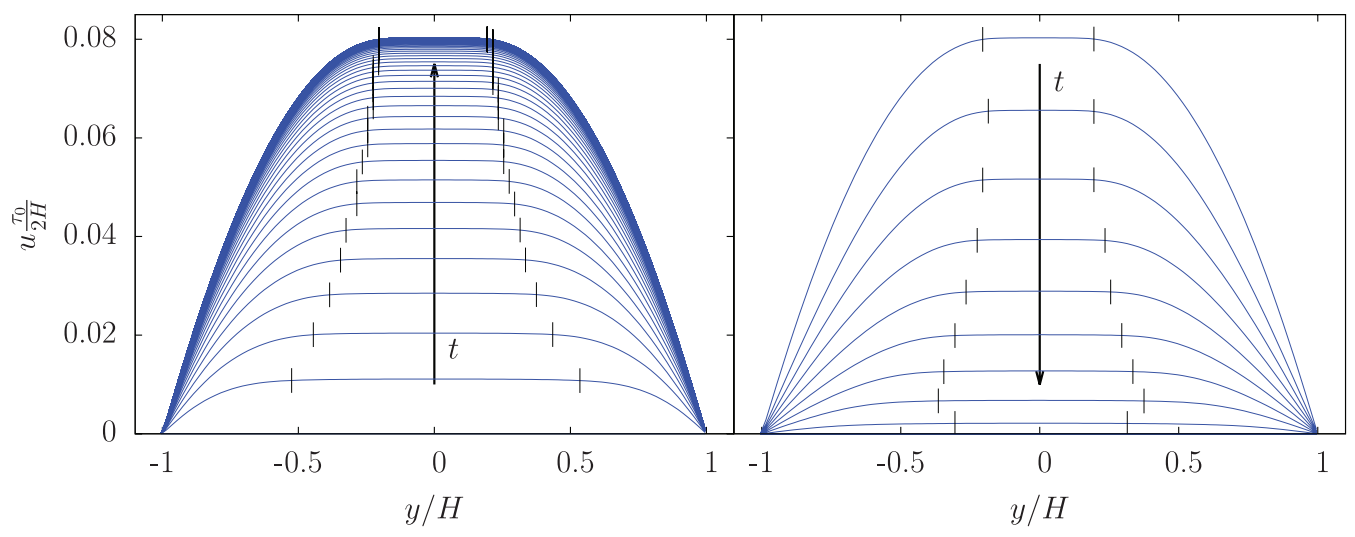

FIG. 7. Temporal evolution of the velocity after startup (left), respectively, cessation (right) of pressure-driven $2 \mathrm{D}$ channel flow, nonlinear Maxwell model with $\theta=10^{2}$. Small vertical lines mark the points where the normalized velocity gradient $\left|\kappa_{x y}(y) / \kappa_{x y}(-H)\right|$ is $5 \%$, as an indicator of the plug width. 


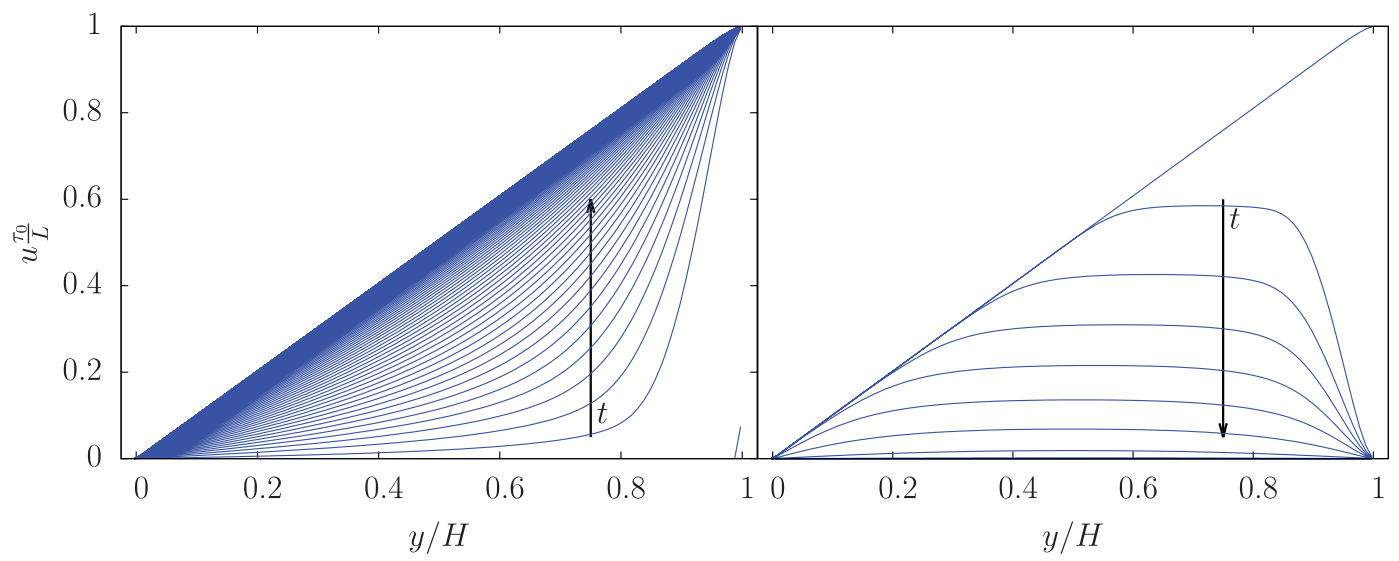

FIG. 8. Velocity profiles of a simple shear flow stopped at $t=0$, for various times $t>0$. Lines are the result of the LB simulation with the nonlinear Maxwell model for $\theta=10^{2}$, with initial wall velocity $u_{0}=10^{-2} L / \tau_{0}$.

\section{CONCLUSIONS}

We propose a tensorial constitutive equation intended to capture certain qualitative features of a microscopically justified ITT-MCT model ${ }^{8}$ for the nonlinear rheology of dense glass-forming colloidal suspensions. This nonlinear generalization of the Maxwell model in particular captures the shear-thinning and yield-stress behavior of glass forming fluids. It is material objective, i.e., its tensorial structure is compatible with the general laws of continuum mechanics and coordinate-frame transformations. The model is simple enough to allow for analytical solutions in certain cases, in particular pressure-driven Poiseuille flow through a planar channel.

We have developed a modified lattice-Boltzmann simulation scheme to address the flow of non-Newtonian fluids including the proper tensorial structure of their constitutive equations. In particular, care has been taken to include non-Newtonian flow-induced pressure differences that arise through normal-stress differences. These pressure differences cause the pressure to rise in the plug at the center of the channel. This pressure variation couples, at the LB level, to a density variation (not unlike the hydrodynamic interaction effect discussed by Nott and Brady ${ }^{72}$ ). Here it has to be noted that the nonlinear Maxwell model we study exhibits rather large normal-stress differences, exaggerating this effect. This may explain why in MD simulations of plug flow of glass-forming fluids ${ }^{15}$ find only a minor cross-channel variation in density.

At present we restrict ourselves to a confirmation of the analytical result in planar flow, where these normal stresses do not couple back to the flow field. But our method is easily applied to cases where flow-density couplings become important and may give rise to nontrivial shear localization. ${ }^{16}$ While the Maxwell relaxation time $\tau$ has been kept constant above, it should, by its microscopic physical motivation, sensitively depend on the local density. We leave this extension for further studies.

The modified LB simulation scheme proposed here is found to give accurate results in the steady-state flow for the shear-thinning model involving a spread in relaxation times as large as a factor $10^{3}$. Larger differences can be handled at the cost of finer grid resolutions and hence computing time. The study of startup flow and cessation of flow demonstrate that also beyond the steady state, the proposed modification of the LB algorithm gives accurate results. In particular, it is capable of reproducing the finite stopping-time singularity that is typical of yield-stress fluids. Here, the finite relaxation time $\tau$ introduced in the Maxwell model serves as a natural regularization parameter.

Other numerical schemes frequently used to simulate non-Newtonian fluid flows include finite-element and finitevolume modeling, either coupled with constitutive-equation solvers (see, e.g., Refs. 73-75), or for the simpler generalizedNewtonian fluids. ${ }^{76}$ Also within these schemes, the inclusion of non-trivial constitutive equations poses subtleties. The LB method is a viable alternative that is computationally efficient.

\section{ACKNOWLEDGMENTS}

We thank M. E. Cates and D. Marenduzzo for fruitful discussions, and M. Fuchs for his valuable suggestions. Th.V. thanks for funding through the Helmholtz-Gemeinschaft, Young Investigator Group HGF VH-NG 406, and DFG Research Unit FOR1394 "Nonlinear Response to Probe Vitrification," project P3. S.P. and Th.V. thank for funding and travel support through the Zukunftskolleg, Universität Konstanz.

\section{APPENDIX: DERIVATION OF THE MODIFIED LB ALGORITHM}

Our LB algorithm differs from previous ones by the choice of the forcing term $F_{i}$, Eq. (8). To clarify this choice, we briefly repeat the main points of the Chapman-Enskog expansion describing the continuum limit of the LB scheme, following the presentation by Dünweg and Ladd. ${ }^{19}$ Introducing a small parameter $\varepsilon$ (the Knudsen number), we set $\tilde{\vec{r}}=\varepsilon \vec{r}$, $\tilde{t}=\varepsilon t$, and $\hat{t}=\varepsilon^{2} t$. We assume an expansion of the distribution functions $n_{i}=n_{i}^{(0)}+\varepsilon n_{i}^{(1)}+\cdots$, and equivalently for $\Delta_{i}$ and $F_{i}$. The LB update can then be discussed on the two time scales of momentum convection and diffusion. To zeroth order in $\varepsilon$, we get $\Delta_{i}^{(0)}=F_{i}^{(0)}=0$; the $n_{i}^{(0)}$ are collision invariants and thus identified with the equilibrium distribution 
$n_{i}^{\text {eq }}$. It should be a function of $\rho$ and $\vec{j}$ only, in order to avoid spurious invariants of the algorithm.

From the first two orders of Eq. (3), one gets

$$
\begin{gathered}
\vec{c}_{i} \cdot \vec{\partial}_{\tilde{r}} n_{i}^{(0)}+\partial_{\tilde{t}} n_{i}^{(0)}=(1 / \delta t) \Delta_{i}^{(1)}+(1 / \delta t) F_{i}^{(1)}, \\
\frac{1}{2}\left[\left(\vec{c}_{i} \cdot \vec{\partial}_{\tilde{r}}\right)+\partial_{\tilde{t}}\right]\left(n_{i}^{(1) *}+n_{i}^{(1)}\right)+\partial_{\hat{t}} n_{i}^{(0)} \\
=(1 / \delta t)\left(\Delta_{i}^{(2)}+F_{i}^{(2)}\right) .
\end{gathered}
$$

Recall now that $\vec{\partial}=\varepsilon \vec{\partial}_{\tilde{r}}$ and $\partial_{t}=\varepsilon \partial_{\tilde{t}}+\varepsilon^{2} \partial_{\hat{t}}$. Summing over all directions, i.e., taking the zeroth velocity-moment of Eqs. [(A1a) and (A1b)], these equations can be combined to give $\partial_{t} \sum_{i}\left(n_{i}^{*}+n_{i}\right) / 2+\vec{\partial} \cdot \sum_{i} \vec{c}_{i}\left(n_{i}^{*}+n_{i}\right) / 2$ $=(\varepsilon / \delta t) \sum_{i}\left(\Delta_{i}^{(1)}+F_{i}^{(1)}\right)+O\left(\varepsilon^{2}\right)$. The continuity equation for the density, Eq. (1), is recovered if the term on the right-hand side vanishes identically (which is the case in standard LB schemes), or can be written as a time-derivative. In order to account for non-Newtonian pressure changes, we adopt the second choice. Specifically, set $(\varepsilon / \delta t) \sum_{i}\left(\Delta_{i}^{(1)}\right.$ $\left.+F_{i}^{(1)}\right)=(\delta \rho(t)-\delta \rho(t-\delta t)) / \delta t=\partial_{t} \delta \rho+O(\varepsilon)$, where $\delta \rho=-\operatorname{tr} \Sigma^{\mathrm{nN}} /\left(c_{s}^{2} d\right)$ is the flow-induced density change. We then define the hydrodynamic density and current as in Eq. (4):

$$
\begin{gathered}
\rho(\vec{r}, t)=\sum_{i} n_{i}^{\mathrm{eq}}=\sum_{i} \frac{n_{i}^{*}(\vec{r}, t)+n_{i}(\vec{r}, t)}{2}-\delta \rho(\vec{r}, t), \\
\vec{J}(\vec{r}, t)=\sum_{i} \vec{c}_{i} n_{i}^{\mathrm{eq}}=\sum_{i} \vec{c}_{i} \frac{n_{i}^{*}(\vec{r}, t)+n_{i}(\vec{r}, t)}{2} .
\end{gathered}
$$

This definition of the density reduces to the standard LB expression $\sum_{i} n_{i}$ in the case of traceless non-Newtonian stresses. As we shall see below, our definition of the momentum current agrees with the standard one used in LB simulations with external force densities, ${ }^{51}$ and it reduces to $\sum_{i} \vec{c}_{i} n_{i}$ in the case of vanishing external force.

Taking the first velocity-moment of Eqs. [(A1a) and (A1b)] results in

$$
\partial_{t} j_{\alpha}+\partial_{\beta} \pi_{\alpha \beta}^{\text {eq }}+\frac{1}{2} \partial_{\beta}\left(\pi_{\alpha \beta}^{\text {neq } *}+\pi_{\alpha \beta}^{\text {neq }}\right)=f_{\alpha}^{\text {ex }},
$$

where we have introduced the second moment of the distribution functions, $\pi_{\alpha \beta}=\sum_{i} c_{i \alpha} c_{i \beta} n_{i}$ and set $n^{\text {neq }}=\varepsilon n^{(1)}$ $+O\left(\varepsilon^{2}\right)$. We have also set $f_{\alpha}^{\text {ex }}=\sum_{i} c_{i \alpha}\left(\Delta_{i}+F_{i}\right) /(\delta t)$, which will be identified with the external body-force density. Equation (A3) shall recover the Navier-Stokes equation, Eq. (2). Since both the equilibrium pressure $p_{0}$ and the Eulerian stresses are given by $\rho$ and $\vec{u}$ alone, they should be connected to the equilibrium contribution in Eq. (A3),

$$
\pi_{\alpha \beta}^{\mathrm{eq}}=p_{0} \delta_{\alpha \beta}+\rho u_{\alpha} u_{\beta} .
$$

Taking Eqs. [(A2a) and (A2b)] and (A4) together, a second-order accurate expression for the LB equilibrium distribution is given by Eq. (5) if the set of $\vec{c}_{i}$ obeys the symme- tries of a cubic lattice and at least three different magnitudes $c_{i}$ are chosen. The D2Q9 model chosen in our work is the simplest model that is compatible with these conditions. The weight coefficients $a^{c_{i}}$ depend only on the velocity magnitudes and obey $\sum_{i} a^{c_{i}}=1$. The construction of $n_{i}^{\text {eq }}$ according to Eq. (5) also implies the speed of sound as $c_{s}=c / \sqrt{3}$, and the linearized equation of state, $p_{0}=\rho c_{s}^{2}$, as mentioned in the main text.

The nonequilibrium part of the $\pi_{\alpha \beta}$ must recover the viscous stresses. By comparison of Eqs. (2) and (A3), $\Sigma_{\alpha \beta}$ $=\left(\pi_{\alpha \beta}^{\text {neq* }}+\pi_{\alpha \beta}^{\text {neq }}\right) / 2$. Making use of the equilibrium distribution function, the second moment of Eq. (A1a),

$$
\partial_{\tilde{t}} \pi_{\alpha \beta}^{(0)}+\partial_{\tilde{\gamma}} \phi_{\alpha \beta \gamma}^{(0)}=(1 / \delta t)\left(\pi_{\alpha \beta}^{(1) *}-\pi_{\alpha \beta}^{(1)}\right),
$$

can be explicitly calculated. Here, $\phi_{\alpha \beta \gamma}$ is the third moment of the LB distribution function. From the conservation laws and the LB equation of state, it follows $\phi_{\alpha \beta \gamma}^{(0)}=\rho c_{s}^{2}\left(u_{\alpha} \delta_{\beta \gamma}\right.$ $\left.+u_{\beta} \delta_{\alpha \gamma}+u_{\gamma} \delta_{\alpha \beta}\right) \quad$ and $\quad \partial_{\tilde{t}} \pi_{\alpha \beta}^{(0)}=-c_{s}^{2}\left(\delta_{\alpha \beta} \partial_{\tilde{\gamma}} j_{\gamma}+u_{\beta} \partial_{\tilde{\alpha}} \rho\right.$ $\left.+u_{\alpha} \partial_{\tilde{\beta}} \rho\right)+\left(u_{\beta} f_{\alpha}^{\operatorname{ex}(1)}+u_{\alpha} f_{\beta}^{\operatorname{ex}(1)}\right)+\left(c_{s}^{2} \delta_{\alpha \beta}-u_{\alpha} u_{\beta}\right)\left(\partial_{\tilde{t}} \delta \rho\right)$, where we have neglected terms of $O\left(M a^{3}\right)$. Taking these results together we arrive at

$$
\begin{aligned}
\pi_{\alpha \beta}^{\text {neq } *}-\pi_{\alpha \beta}^{\mathrm{neq}}= & (\delta t) \rho c_{s}^{2}\left(\partial_{\alpha} u_{\beta}+\partial_{\beta} u_{\alpha}\right) \\
& +(\delta t)\left(u_{\alpha} f_{\beta}^{\mathrm{ex}}+u_{\beta} f_{\alpha}^{\mathrm{ex}}\right) \\
& +\left(c_{s}^{2} \delta_{\alpha \beta}-u_{\alpha} u_{\beta}\right)(\delta t)\left(\partial_{t} \delta \rho\right) .
\end{aligned}
$$

The approximations involved in deriving this equation imply that the LB method provides an approximation of the deviatoric stress tensor that is second order in the lattice parameters, although this can be spoiled by the boundary conditions. ${ }^{55,56}$

To proceed further, assume a collision operator of the form $\Delta_{i}=\mathcal{L}_{i j} n_{j}^{\text {neq }}$. To keep the derivation simple, we restrict ourselves to the single-relaxation-time BGK model: $\mathcal{L}_{i j}=-\delta_{i j} / \tau_{\mathrm{LB}}, \quad$ so $\quad$ that $\pi_{\alpha \beta}^{\text {neq } *}-\pi_{\alpha \beta}^{\text {neq }}=-\left(1 / \tau_{\mathrm{LB}}\right) \pi_{\alpha \beta}^{\text {neq }}$ $+\mathcal{F}_{\alpha \beta}$, where $\mathcal{F}_{\alpha \beta}=\sum_{i} c_{i \alpha} c_{i \beta} F_{i}$ is the second velocitymoment of the LB forcing term. Combining this with Eq. (A6) to evaluate $\pi_{\alpha \beta}^{\text {neq* }}+\pi_{\alpha \beta}^{\text {neq }}=\left(1-2 \tau_{\mathrm{LB}}\right)\left(\pi_{\alpha \beta}^{\text {neq* }}\right.$ $\left.-\pi_{\alpha \beta}^{\text {neq }}\right)+2 \tau_{\mathrm{LB}} \mathcal{F}_{\alpha \beta}$, we can by comparison of Eqs. (2) and (A3) identify the viscous stresses: from the first term in Eq. (A6), we get the Newtonian stresses, and $\mathcal{F}_{\alpha \beta}$ is still at our disposal to incorporate non-Newtonian stresses. However, Eq. (A6) contains spurious terms involving $\vec{f}^{\text {ex }}$ and $\delta \rho$. To cancel them, $\mathcal{F}_{\alpha \beta}$ must contain matching terms, leaving us with

$$
\begin{aligned}
\mathcal{F}_{\alpha \beta}= & \left(1-\frac{1}{2 \tau_{\mathrm{LB}}}\right)(\delta t)\left(u_{\alpha} f_{\beta}^{\mathrm{ex}}+u_{\beta} f_{\alpha}^{\mathrm{ex}}\right) \\
& +\left(1-\frac{1}{2 \tau_{\mathrm{LB}}}\right)(\delta t)\left(c_{s}^{2} \delta_{\alpha \beta}-u_{\alpha} u_{\beta}\right)\left(\partial_{t} \delta \rho\right)-\frac{1}{\tau_{\mathrm{LB}}} \bar{\Sigma}_{\alpha \beta}^{\mathrm{nN}} .
\end{aligned}
$$

Equation (A7) is a condition on the second moments of the LB forcing term. From our definitions of $f_{\alpha}^{\mathrm{ex}}$ and $\partial_{t} \delta \rho$, we get further conditions on the first and zeroth velocitymoments of $F_{i}$, viz. $\sum_{i} c_{i \alpha} F_{i}=(\delta t) f_{\alpha}^{\mathrm{ex}}\left(1-1 /\left(2 \tau_{\mathrm{LB}}\right)\right)$ and $\sum_{i} F_{i}=(\delta t)\left(\partial_{t} \delta \rho\right)\left(1-1 /\left(2 \tau_{\mathrm{LB}}\right)\right)$. This allows to construct $F_{i}$ 
with second-order accuracy, and leads to the expression given in Eq. (8).

As a cross-check, imagine a LB simulation with $\tau_{\mathrm{LB}}$ set up according to some Newtonian viscosity $\eta$; and set $\Sigma_{\alpha \beta}^{\mathrm{nN}}=\Delta \eta\left(\partial_{\alpha} u_{\beta}+\partial_{\beta} u_{\alpha}\right)$ to be of Newtonian form again. Reconstructing $n_{i}^{\text {neq }}$ up to second order through its known first moments, direct inspection reveals $\Delta_{i}+F_{i}=-\left(1 / \tau_{\mathrm{LB}}^{\prime}\right) n_{i}^{\text {neq }}$, where $\tau_{\mathrm{LB}}^{\prime}$ corresponds to Eq. (7) with $\eta \mapsto \eta+\Delta \eta$. Our forcing term thus reduces for purely Newtonian stresses to the standard BGK form.

${ }^{1}$ R. G. Larson, The Structure and Rheology of Complex Fluids (Oxford University Press, Oxford, 1998).

${ }^{2}$ P. Oswald, Rheophysics (Cambridge University Press, Cambridge, 2009).

${ }^{3}$ R. B. Bird, R. C. Armstrong, and O. Hassager, Dynamics of Polymeric Liquids (John Wiley and Sons, York, 1987), Vol. 1.

${ }^{4} \mathrm{M}$. Doi and S. F. Edwards, The Theory of Polymer Dynamics (Oxford University Press, Oxford, 1989).

${ }^{5}$ M. Fuchs and M. E. Cates, Phys. Rev. Lett. 89, 248304 (2002).

${ }^{6}$ J. M. Brader, Th. Voigtmann, M. E. Cates, and M. Fuchs, Phys. Rev. Lett. 98, 058301 (2007).

${ }^{7}$ J. M. Brader, M. E. Cates, and M. Fuchs, Phys. Rev. Lett. 101, 138301 (2008).

${ }^{8}$ J. M. Brader, Th. Voigtmann, M. Fuchs, R. G. Larson, and M. E. Cates, Proc. Natl. Acad. Sci. USA 106, 15186 (2009).

${ }^{9}$ Th. Voigtmann, J. M. Brader, M. Fuchs, and M. E. Cates, Soft Matter 8, 4244 (2012)

${ }^{10}$ M. Fuchs and M. E. Cates, Faraday Discuss. 123, 267 (2003).

${ }^{11}$ M. Fuchs and M. Ballauff, Coll. Surf. A 270-271, 232 (2005).

${ }^{12}$ Th. Voigtmann, Eur. Phys. J. E 34, 106 (2011).

${ }^{13}$ M. Siebenbürger, M. Ballauff, and Th. Voigtmann, Phys. Rev. Lett. 108, 255701 (2012).

${ }^{14}$ Th. Voigtmann, AIP Conf. Proc. 1518, 94 (2013).

${ }^{15}$ F. Varnik and D. Raabe, Phys. Rev. E 77, 011504 (2008).

${ }^{16}$ R. Besseling, L. Isa, P. Ballesta, G. Petekidis, M. E. Cates, and W. C. K. Poon, Phys. Rev. Lett. 105, 268301 (2010).

${ }^{17}$ S. Succi, The Lattice Boltzmann Equation for Fluid Dynamics and Beyond (Oxford University Press, Oxford, 2001).

${ }^{18}$ R. Benzi, S. Succi, and M. Vergassola, Phys. Rep. 222, 145 (1992).

${ }^{19}$ B. Dünweg and A. J. C. Ladd, Adv. Polym. Sci. 221, 89 (2009).

${ }^{20}$ C. K. Aidun and J. R. Clausen, Annu. Rev. Fluid Mech. 42, 439 (2010).

${ }^{21}$ E. Aharonov and D. H. Rothman, Geophys. Res. Lett. 20, 679, doi:10.1029/93GL00473 (1993).

${ }^{22}$ S. Gabbanelli, G. Drazer, and J. Koplik, Phys. Rev. E 72, 046312 (2005).

${ }^{23}$ J. Boyd, J. Buick, and S. Green, J. Phys. A 39, 14241 (2006).

${ }^{24}$ D. Kehrwald, J. Stat. Phys. 121, 223 (2005).

${ }^{25}$ R. Ouared and B. Chopard, J. Stat. Phys. 121, 209 (2005).

${ }^{26}$ J. Boyd, J. M. Buick, and S. Green, Phys. Fluids 19, 093103 (2007).

${ }^{27}$ Y. Yan and J. Koplik, Phys. Rev. E 77, 036315 (2008).

${ }^{28}$ M. Ashrafizaadeh and H. Bakhshaei, Comput. Math. Appl. 58, 1045 (2009).

${ }^{29}$ D. Wang and J. Bernsdorf, Comput. Math. Appl. 58, 1030 (2009).

${ }^{30}$ G. H. Tang, P. X. Ye, and W. Q. Tao, J. Non-Newton. Fluid Mech. 165, 1536 (2010)

${ }^{31}$ A. Vikhansky, J. Non-Newton. Fluid Mech. 155, 95 (2008).

${ }^{32}$ T. N. Phillips and G. W. Roberts, IMA J. Appl. Math. 76, 790 (2011).

${ }^{33}$ G. Pontrelli, S. Ubertini, and S. Succi, J. Stat. Mech. (2009) P06005.

${ }^{34}$ J. J. Derksen and Prashant, J. Non-Newton. Fluid Mech. 160, 65 (2009).
${ }^{35}$ J. J. Derksen, Phys. Fluids 21, 083302 (2009).

${ }^{36}$ M. Junk, J. Comput. Phys. 151, 947 (1999).

${ }^{37}$ M. Junk and S. V. R. Rao, J. Comput. Phys. 155, 178 (1999).

${ }^{38}$ T. Inamuro, Philos. Trans. R. Soc., London A 360, 477 (2002).

${ }^{39}$ C. Denniston, E. Orlandini, and J. M. Yeomans, Phys. Rev. E 63, 056702 (2001).

${ }^{40}$ N. Sulaiman, D. Marenduzzo, and J. M. Yeomans, Phys. Rev. E 74, 041708 (2006).

${ }^{41}$ O. Malaspinas, N. Fiétier, and M. Deville, J. Non-Newton. Fluid Mech. 165, 1637 (2010).

${ }^{42}$ D. Marenduzzo, E. Orlandini, M. E. Cates, and J. M. Yeomans, Phys. Rev. E 76, 031921 (2007).

${ }^{43}$ O. Henrich, D. Marenduzzo, K. Stratford, and M. E. Cates, Comput. Math. Appl. 59, 2360 (2010).

${ }^{44}$ G. N. Frantziskonis, Phys. Rev. E 83, 066703 (2011).

${ }^{45}$ R. Benzi, M. Bernaschi, M. Sbragaglia, and S. Succi, EPL 91, 14003 (2010).

${ }^{46}$ M. Sbragaglia, R. Benzi, M. Bernaschi, and S. Succi, Soft Matter 8, 10773 (2012).

${ }^{47}$ R. Benzi, M. Bernaschi, M. Sbragaglia, and S. Succi, EPL 104, 48006 (2013).

${ }^{48}$ H. Chen, S. Kandasamy, S. Orszag, R. Shock, S. Succi, and V. Yakhot, Science 301, 633 (2003).

${ }^{49}$ A. J. C. Ladd and R. Verberg, J. Stat. Phys. 104, 1191 (2001).

${ }^{50}$ R. W. Nash, R. Adhikari, and M. E. Cates, Phys. Rev. E 77, 026709 (2008).

${ }^{51}$ Z. Guo, C. Zheng, and B. Shi, Phys. Rev. E 65, 046308 (2002).

${ }^{52}$ S. H. Kim and H. Pitsch, Phys. Fluids 19, 108101 (2007).

${ }^{53}$ Q. Zou and X. He, Phys. Fluids 9, 1591 (1997).

${ }^{54}$ D. J. Holdych, D. R. Noble, J. G. Georgiadis, and R. O. Buckius, J. Comput. Phys. 193, 595 (2004).

${ }^{55}$ T. Krüger, F. Varnik, and D. Raabe, Phys. Rev. E 79, 046704 (2009).

${ }^{56}$ T. Krüger, F. Varnik, and D. Raabe, Phys. Rev. E 82, 025701(R) (2010).

${ }^{57}$ Palabos V1.1r0, see http://www.palabos.org.

${ }^{58}$ K. Suzuki and H. Hayakawa, Phys. Rev. E 87, 012304 (2013).

${ }^{59}$ J. G. Oldroyd, Proc. R. Soc. London A 200, 523 (1950).

${ }^{60}$ J. L. White and A. B. Metzner, J. Appl. Polym. Sci. 7, 1867 (1963).

${ }^{61}$ C. Verdier and D. D. Joseph, J. Non-Newton. Fluid Mech. 31, 325 (1989).

${ }^{62}$ C. Derec, A. Ajdari, and F. Lequeux, Eur. Phys. J. E 4, 355 (2001).

${ }^{63}$ P. Coussot, Q. D. Nguyen, H. T. Huynh, and D. Bonn, Phys. Rev. Lett. 88, 175501 (2002).

${ }^{64}$ S. M. Fielding, R. G. Larson, and M. E. Cates, Phys. Rev. Lett. 108, 048301 (2012).

${ }^{65}$ S. Papenkort, "Flow of yield-stress fluids through channels: Lattice Boltzmann simulations," Ph.D. thesis, Universität Konstanz, Germany, 2013.

${ }^{66}$ R. R. Huilgol, Phys. Fluids 14, 1269 (2002).

${ }^{67}$ R. R. Huilgol, B. Mena, and J. M. Piau, J. Non-Newton. Fluid Mech. 102, 97 (2002).

${ }^{68}$ S. Mandal, M. Gross, D. Raabe, and F. Varnik, Phys. Rev. Lett. 108, 098301 (2012).

${ }^{69}$ M. Chatzimina, G. C. Georgiou, I. Argyropaidas, E. Mitsoulis, and R. R. Huilgol, J. Non-Newton. Fluid Mech. 129, 117 (2005).

${ }^{70}$ G. K. Batchelor, An Introduction to Fluid Dynamics (Cambridge University Press, Cambridge, 1967).

${ }^{71}$ L. Muravleva, E. Muravleva, G. C. Georgiou, and E. Mitsoulis, J. Non-Newton. Fluid Mech. 165, 544 (2010).

${ }^{72}$ P. R. Nott and J. F. Brady, J. Fluid Mech. 275, 157 (1994).

${ }^{73}$ D. S. Malkus, Commun. Appl. Numer. Math. 1, 275 (1985).

${ }^{74}$ P. Saramito, J. Non-Newton. Fluid Mech. 60, 199 (1995).

${ }^{75}$ E. J. Dean, R. Glowinski, and G. Guidoboni, J. Non-Newton. Fluid Mech. 142, 36 (2007).

${ }^{76}$ J. Hron, J. Málek, and S. Turek, Int. J. Numer. Meth. Fluids 32, 863 (2000). 